\title{
Impact of a nitrogen emission control area (NECA) on the future air quality and nitrogen deposition to seawater in the Baltic Sea region
}

Matthias Karl et al.

Correspondence to: Matthias Karl (matthias.karl@hzg.de)

The copyright of individual parts of the supplement might differ from the CC BY 4.0 License. 


\section{S1. Methodology and results for the evaluation of modelled precipitation}

The precipitation amount and frequency from COSMO-CLM output was compared against daily precipitation measurements from rain gauge stations operated by the Swedish Meteorological and Hydrological Institute (SMHI). 1804 precipitation stations in Sweden were recording daily precipitation sums during 2012; each measurement starting at 6 a.m. UTC on the previous day until 6 a.m. UTC of the current day and is available from the SMHI opendata portal (http://opendata-catalog.smhi.se/explore/). The station network for daily precipitation collection densely covers the regions of south and middle Sweden (Götaland and Svealand) where each station represents an area of about $10 \times 10 \mathrm{~km}^{2}$, with lower density of stations in the northern part of Sweden (Södra Norrland and Norra Norrland). Precipitation measurements are known to suffer from evaporation and wind loss, especially on days with very low rain rates (Nespor and Sevruk, 1998). Therefore, a precipitation threshold of $1 \mathrm{~mm} \mathrm{~d}^{-1}$ is commonly used for the definition of dry days (WMO, 2011). In the present comparison of modelled with observed precipitation no observational threshold was applied in the data analysis. However, in order to determine the performance of COSMO-CLM with respect to the prediction of the number of days without rainfall and hence no wet deposition is examined based on the definition of dry days by a threshold of $0.1 \mathrm{~mm} \mathrm{~d}^{-1}$ for the model and observational data.

Note for the comparison of modelled and observed precipitation, that the model data is a grid average (either of grid boxes with 0.11 degree or 0.025 degree cell width) whereas the rain gauge data represent a point measurement. Hence, the high-resolution output of COSMOCLM should better capture the variability of the precipitation measurements. The modelobservation comparison was done for the three different configurations of COSMO-CLM: 0.11 degree grid resolution with Tiedtke scheme for convection (“011”), 0.025 degree grid resolution with Tiedtke scheme for convection ("0025_Tiedtke"), and 0.025 degree grid resolution with convection-permitting configuration (“0025_convper”). Fig. S1 shows the 
monthly precipitation amounts from the three model configurations of the summer months for the Baltic Sea and North Sea region and compares to the measured precipitation amount at the Swedish rain gauge stations (circles filled with colour indicating the observed value).

Fig. S2 shows the probability distributions of the differences in seasonal averaged (winter months and summer months of 2012) daily precipitation sums from the three model outputs and the observation data in the four regions of Sweden. The percentage fraction of days with zero difference between model and observation and days with difference of $0.1 \mathrm{~mm} \mathrm{~d}^{-1}$ (“delta01" days), corresponding to the threshold value, was calculated. Large deviations between model and observations $\left(>10 \mathrm{~mm} \mathrm{~d}^{-1}\right)$ are rare for all three configurations. For “0025_convper", the percentage fraction of days with model-observation deviations below the threshold ("delta01" days) is in the range of $18-34 \%$ in winter and $29-38 \%$ in summer, depending on the region.

The fraction of observed dry days (daily sum $<0.1 \mathrm{~mm} \mathrm{~d}^{-1}$ ), as average of all stations of one region, of summer and winter (Table S1) is always higher than the fraction of "delta01" days in the corresponding model data. This implies that COSMO-CLM, on a statistical average, predicts more precipitation days than observed. For "0025_convper", the number of precipitation days, depending on the region, is 9-18\% higher in summer and 9-11\% higher in winter than observed (assuming that the "delta01" days correspond to dry days).

By summing up the model-observation differences of all days in summer and winter averaged over all stations of one region, the model bias for precipitation amounts was determined (Table S2). Note that in this study winter is defined as JFD, including the months January, February and December from 2012 since the simulation was only done for one year. In summer, precipitation in "0025_convper" has a relative bias of $-25 \%,-19 \%, 51 \%$, and $42 \%$ in Götaland, Svealand, S. Norland, N. Norland, respectively, compared to observed 
precipitation amounts. In winter, precipitation from "0025_convper" has a much lower relative bias $(-4 \%,-7 \%, 3 \%$, and $32 \%$ in Götaland, Svealand, S. Norland, N. Norland, respectively).

The probability distribution of differences for all months for Götaland is shown in Fig. S3. The convection-permitting configuration "0025_convper" reduces the frequency of negative differences in the range of $0.1-4 \mathrm{~mm} \mathrm{~d}^{-1}$ for daily rainfall in June compared to “0025_Tiedtke”. The frequencies of negative differences between observation and model is similar for all summer months, while observed total monthly precipitation amount is highest in June (Götaland station average, June: $111 \mathrm{~mm}$, July: $87 \mathrm{~mm}$, August: $68 \mathrm{~mm}$ ). COSMOCLM performs better in the cold season (from October to March) in Götaland when differences between observations and model are in the range between -4 and $+6 \mathrm{~mm} \mathrm{~d}^{-1}$ for more than $90 \%$ of the time (Fig. S3). 


\section{S2. Methodology and results for the evaluation of modelled wet deposition of nitrogen}

The modelled wet deposition of nitrogen from CMAQ output was compared against data from the EMEP monitoring programme (Tørseth et al., 2012; EMEP, 2014). Observation data was obtained from the EBAS database (http://ebas.nilu.no/). Modelled wet deposition of nitrogen was separately compared for nitrate, $\mathrm{NO}_{3}{ }^{-}\left(\mathrm{WNO}_{3}\right)$, representing oxidised nitrogen and modelled wet deposition of ammonium, $\mathrm{NH}_{4}{ }^{+}\left(\mathrm{WNH}_{4}\right)$, representing reduced nitrogen. Modelled wet deposition of $\mathrm{HNO}_{3}$ was included in $\mathrm{WNO}_{3}$ and modelled wet deposition of $\mathrm{NH}_{3}$ was included in $\mathrm{WNH}_{4}$, because it is assumed that the gases are partially or fully dissolved in the sampled rainwater. The summation also gives a more robust estimate of the wet deposition of oxidised and reduced nitrogen. Measured concentrations of $\mathrm{NO}_{3}{ }^{-}$and $\mathrm{NH}_{4}{ }^{+}$ in rainwater were converted into nitrogen deposition per area by the amount of rainwater measured at the respective station. Daily sums of wet deposition were calculated from the volume-weighted concentrations multiplied by the precipitation amounts. The comparison of the daily sum of wet deposition was done in terms of mean values ( $\mu_{\text {Mod }}$ and $\left.\mu_{\mathrm{Obs}}\right)$, the Spearman's correlation coefficient $\left(\mathrm{R}_{\mathrm{Spr}}\right)$ and the normalized mean bias $(\mathrm{NMB})$. Only days with predicted and observed rain events in common were included in the comparison. Several stations in the Baltic Sea region had only few measurements during the period. Stations with less than seven model-observation pairs were excluded from the statistical analysis. CMAQ model data from the intermediate grid (CD16) and from the high-resolution grid (CD04) were evaluated separately.

Plots in Fig. 3b-g show the time series modelled and observed daily sums of $\mathrm{WNO}_{3}$ at selected stations, while all other stations are shown in Fig. S4. $\mathrm{WNO}_{3}$ is underestimated at all stations included in the statistical analysis (Table S3), most severely at the Finnish stations and at Zingst (NMB between -0.75 and -0.90 ). 
$\mathrm{WNH}_{4}$ is underestimated at all stations included in the statistical analysis (Table S4; corresponding time series are plotted in Fig. S5). The underestimation is highest for Zingst and the Finnish stations, as for $\mathrm{WNO}_{3}$.

To account for the fact that the days with predicted rain often do not correspond to days with observed rain, seasonal averages (spring, summer and autumn) were calculated for $\mathrm{WNO}_{3}$ (Table S5) and $\mathrm{WNH}_{4}$ (Table S6) independently for CD04 model data and observation data. The joint underestimation of $\mathrm{WNO}_{3}$ and $\mathrm{WNH}_{4}$ at Zingst and the Finnish stations is confirmed in this analysis. At Zingst, seasonal averages of $\mathrm{WNO}_{3}$ based on model data are lower by $-52 \%$ to $-63 \%$ and seasonal averages of $\mathrm{WNH}_{4}$ based on model data are lower by $64 \%$ to $-79 \%$ than the corresponding seasonal averages based on observations. The ratio of modelled to observed seasonal average of $\mathrm{WNO}_{3}$ shows little variation for the three seasons (0.37-0.48). At Preila, a coastal station in Lithuania, both observed averages of $\mathrm{WNO}_{3}$ and $\mathrm{WNH}_{4}$ are underestimated in spring and autumn, but not in summer. The ratio of modelled to observed seasonal averages of $\mathrm{WNO}_{3}$ and $\mathrm{WNH}_{4}$ show a consistent seasonal pattern at the nine stations (Fig. S6), pointing to the formation and atmospheric transport of particulate ammonium nitrate as common cause. 


\section{S3. Methodology and results for the evaluation of modelled air pollutant concentrations}

The modelled surface air concentrations of $\mathrm{O}_{3}, \mathrm{NO}_{2}, \mathrm{SO}_{2}$ and $\mathrm{PM}_{2.5}$ from CMAQ output of the 4-km resolution grid were compared against measurements at regional background stations of the EMEP monitoring programme available from the EBAS database. In addition to the statistical indicators used in the evaluation of deposition, the root mean square error of the modelled values (RMSE) was included in the evaluation of air concentrations, a frequently used measure of the differences between values predicted by a model and the values actually observed. The evaluation was done for the entire year 2012 and separately for summer (JJA) 2012. In the context of this evaluation of predicted air pollutant concentrations, a correlation coefficient of more than 0.5 was considered to indicate a correlation between modelled and observed time series, while values of 0.7 and above was considered as a good correlation.

The results for the statistical evaluation of modelled daily mean $\mathrm{O}_{3}$ concentrations are summarized in Table S7. Modelled daily means of $\mathrm{O}_{3}$ are in good agreement with measurements at all stations $\left(\mathrm{R}_{\mathrm{Spr}}=0.75, \mathrm{RMSE}=6.8 \mathrm{ppbv}\right.$, both as average of all stations; NMB range: -0.16 to -0.02 ) when the entire year is considered. In summer, ozone is slightly underestimated at the stations in the southern part of the Baltic Sea region (NMB range: -0.23 to -0.12$)$. The overall agreement in summer is, however, fairly good $\left(\mathrm{R}_{\mathrm{Spr}}=0.62, \mathrm{RMSE}=6.9\right.$ ppbv; each as average of all stations).

The evaluation of modelled $\mathrm{NO}_{2}$ based on daily concentrations for the entire year and for summer (Table S8) indicates a better performance of CMAQ over the entire year than over summer alone. A good correlation is obtained at 9 out of 12 stations for the entire year, whereas only 5 stations show a good correlation in summer. MNB is positive at 11 stations for the entire year and at 8 stations for summer. The CD04 simulations predict slightly higher $\mathrm{NO}_{2}$ concentrations than observed at most stations (NMB range: $-0.28-0.44$ for the year; 
NMB range: $-0.31-0.83$ for JJA; average of all stations). RMSE is in the range 1.0-3.2 ppbv for the year and in the range $0.2-3.5 \mathrm{ppbv}$ for summer.

Simulated $\mathrm{SO}_{2}$ daily mean concentrations are correlated with the observed daily mean concentrations at all stations of the Baltic Sea region for the entire year but in summer they are not correlated at several stations (Table S9). The associated RMSE is relatively high $(\mathrm{RMSE}=1.01 \mathrm{ppbv}$ for the year, RMSE $=0.41 \mathrm{ppbv}$ for JJA; average of all stations $)$ but the summer RMSE is lower than in the multi-model study by Giordano et al. (2015) (RMSE range: $2.17-2.34 \mathrm{ppbv}$ ). Observed $\mathrm{SO}_{2}$ concentrations are generally overestimated (NMB range: $-0.04-1.62$ for the year; NMB range: $-0.07-1.84$ for JJA). In particular, summer mean $\mathrm{SO}_{2}$ at the remote stations Ähtäri II (Finland) and Preila (Lithuania) is overestimated by a factor of 2-3.

Data from eight stations was available for the comparison of modelled against observed $\mathrm{PM}_{2.5}$ (Table S10). For the entire year CMAQ performs quite well in the prediction of daily mean $\mathrm{PM}_{2.5}\left(\mathrm{R}_{\mathrm{Spr}}=0.57, \mathrm{NMB}=-0.22, \mathrm{RMSE}=5.6 \mu \mathrm{g} \mathrm{m}-3 ;\right.$ each as average of all stations $)$. In the summer period, $\mathrm{PM}_{2.5}$ is underestimated at all stations (average $\mathrm{NMB}=-0.60$ ). 
Table S1: Percentage fraction of dry days, as observed and as predicted by COSMO-CLM model with configuration "0025_convper" for summer (JJA) and for winter (JFD) of 2012 in the four regions of Sweden. Values are given as average of all stations in a region. For the fraction of predicted dry days it is assumed that the "delta01" days (days with modelobservation difference below the threshold of $0.1 \mathrm{~mm} \mathrm{~d}^{-1}$ ) correspond to dry days.

\begin{tabular}{|c|c|c|c|c|}
\hline \multirow{2}{*}{ Region } & \multicolumn{2}{|c|}{ Summer } & \multicolumn{2}{|c|}{ Winter } \\
\hline & $\begin{array}{c}\text { Predicted } \\
\text { dry days [\%] }\end{array}$ & $\begin{array}{c}\text { Observed } \\
\text { dry days [\%] }\end{array}$ & $\begin{array}{c}\text { Predicted } \\
\text { dry days [\%] }\end{array}$ & $\begin{array}{c}\text { Observed } \\
\text { dry days [\%] }\end{array}$ \\
\hline Götaland & 38 & 47 & 34 & 43 \\
\hline Svealand & 29 & 41 & 32 & 42 \\
\hline S. Norrland & 31 & 47 & 23 & 34 \\
\hline N. Norrland & 31 & 49 & 18 & 28 \\
\hline
\end{tabular}

Table S2: Precipitation bias, calculated as difference between model and observation (M-O; in $\mathrm{mm}$ ) for summer (JJA) and for winter (JFD) of 2012. Values are given as average of all stations in a region.

\begin{tabular}{l||ccc||ccc}
\hline \multirow{2}{*}{\multicolumn{1}{l||}{ Region }} & \multicolumn{3}{c||}{$\begin{array}{c}\text { Precipitation bias (M-O) } \\
\text { [mm] in summer }\end{array}$} & \multicolumn{3}{c}{$\begin{array}{c}\text { Precipitation bias (M-O) } \\
\text { [mm] in winter }\end{array}$} \\
\cline { 2 - 7 } & 011 & $\begin{array}{c}0025_{-} \\
\text {Tiedtke }\end{array}$ & $\begin{array}{c}0025_{-} \\
\text {convper }\end{array}$ & 011 & $\begin{array}{c}0025_{-} \\
\text {Tiedtke }\end{array}$ & $\begin{array}{c}0025_{-} \\
\text {convper }\end{array}$ \\
\hline \hline Götaland & -99 & -35 & -67 & -1 & 0 & -8 \\
\hline Svealand & -83 & -40 & -61 & 20 & -4 & -11 \\
\hline S. Norrland & 10 & 111 & 132 & 50 & 18 & 5 \\
\hline N. Norrland & 49 & 89 & 101 & 43 & 34 & 53 \\
\hline \hline
\end{tabular}


Table S3: Statistical comparison of daily sums of $\mathrm{WNO}_{3}$ for stations of the EMEP monitoring network in the Baltic Sea region. CMAQ model results for the CD16 and CD04 grid domains are evaluated separately. Statistical indicators include mean values of model ( $\left.\mu_{\text {Mod }}\right)$, mean values of observations ( $\left.\mu_{\mathrm{obs}}\right)$, Spearman's correlation coefficient $\left(\mathrm{R}_{\mathrm{Spr}}\right)$, and normalized mean bias (NMB). Unit of mean values is $m g(N) \mathrm{m}^{-2} \mathrm{~d}^{-1} . N$ is the number of samples where precipitation occurred both in the simulation and in the observation. Only stations with more than 7 samples were considered (for station Hailuoto II it is less than 7 for the CD04 grid).

\begin{tabular}{|c|c|c|c|c|c|c|c|c|c|c|}
\hline \multirow{2}{*}{$\begin{array}{l}\text { Station, } \\
\text { Code }\end{array}$} & \multicolumn{5}{|c|}{ CD16 } & \multicolumn{5}{|c|}{ CD04 } \\
\hline & $N$ & $\mathrm{R}_{\mathrm{Spr}}$ & $\mu_{\mathrm{Mod}}$ & $\mu_{\mathrm{obs}}$ & NMB & $N$ & $\mathrm{R}_{\mathrm{Spr}}$ & $\mu_{\text {Mod }}$ & $\mu_{\mathrm{obs}}$ & NMB \\
\hline $\begin{array}{l}\text { Zingst, } \\
\text { DE0009R }\end{array}$ & 14 & -0.09 & 0.65 & 5.23 & -0.88 & 9 & 0.08 & 1.21 & 6.03 & -0.80 \\
\hline $\begin{array}{l}\text { Råö } \\
\text { SE0014R }\end{array}$ & 86 & 0.49 & 2.37 & 3.21 & -0.26 & 77 & 0.55 & 1.76 & 2.95 & -0.40 \\
\hline $\begin{array}{l}\text { Leba, } \\
\text { PL0004R }\end{array}$ & 72 & 0.26 & 1.56 & 3.41 & -0.54 & 58 & 0.40 & 1.48 & 3.78 & -0.61 \\
\hline $\begin{array}{l}\text { Diabla Gora, } \\
\text { PL0005R }\end{array}$ & 75 & 0.29 & 1.25 & 2.42 & -0.48 & 63 & 0.01 & 1.61 & 3.02 & -0.47 \\
\hline $\begin{array}{l}\text { Ähtäri, } \\
\text { FI0004R }\end{array}$ & 12 & 0.06 & 0.75 & 3.57 & -0.79 & 11 & 0.81 & 0.78 & 3.16 & -0.75 \\
\hline $\begin{array}{l}\text { Virolahti II } \\
\text { FI0017R }\end{array}$ & 17 & -0.01 & 0.92 & 5.93 & -0.85 & 14 & -0.17 & 1.07 & 5.42 & -0.80 \\
\hline $\begin{array}{l}\text { Hailuoto II, } \\
\text { FI0053R }\end{array}$ & 13 & 0.32 & 0.69 & 3.07 & -0.78 & 5 & - & - & - & - \\
\hline $\begin{array}{l}\text { Lahemaa, } \\
\text { EE0009R }\end{array}$ & 70 & 0.31 & 1.05 & 1.66 & -0.37 & 50 & 0.27 & 1.10 & 1.84 & -0.40 \\
\hline $\begin{array}{l}\text { Preila } \\
\text { LT0015R }\end{array}$ & 58 & 0.32 & 1.46 & 2.66 & -0.45 & 44 & 0.40 & 2.04 & 2.733 & -0.25 \\
\hline
\end{tabular}


Table S4: Statistical comparison of daily sums of $\mathrm{WNH}_{4}$ for stations of the EMEP monitoring network in the Baltic Sea region. CMAQ model results for the CD16 and CD04 grid domains are evaluated separately. Statistical indicators include mean values of model ( $\left.\mu_{\text {Mod }}\right)$, mean values of observations ( $\left.\mu_{\mathrm{obs}}\right)$, Spearman's correlation coefficient $\left(\mathrm{R}_{\mathrm{Spr}}\right)$, and normalized mean bias (NMB). Unit of mean values is $\operatorname{mg}(\mathrm{N}) \mathrm{m}^{-2} \mathrm{~d}^{-1} . N$ is the number of samples where precipitation occurred both in the simulation and in the observation. Only stations with more than 7 samples were considered (for station Hailuoto II it is less than 7 for the CD04 grid).

\begin{tabular}{|c|c|c|c|c|c|c|c|c|c|c|}
\hline \multirow{2}{*}{$\begin{array}{l}\text { Station, } \\
\text { Code }\end{array}$} & \multicolumn{5}{|c|}{ CD16 } & \multicolumn{5}{|c|}{ CD04 } \\
\hline & $N$ & $\mathrm{R}_{\mathrm{Spr}}$ & $\mu_{\mathrm{Mod}}$ & $\mu_{\mathrm{obs}}$ & NMB & $N$ & $\mathrm{R}_{\mathrm{Spr}}$ & $\mu_{\text {Mod }}$ & $\mu_{\mathrm{obs}}$ & NMB \\
\hline $\begin{array}{l}\text { Zingst, } \\
\text { DE0009R }\end{array}$ & 12 & 0.23 & 0.76 & 8.88 & -0.91 & 8 & 0.52 & 1.54 & 9.60 & -0.84 \\
\hline $\begin{array}{l}\text { Råö } \\
\text { SE0014R }\end{array}$ & 81 & 0.52 & 2.03 & 3.28 & -0.38 & 63 & 0.38 & 1.83 & 3.42 & -0.46 \\
\hline $\begin{array}{l}\text { Leba, } \\
\text { PL0004R }\end{array}$ & 69 & 0.35 & 1.31 & 4.21 & -0.69 & 57 & 0.24 & 1.29 & 4.64 & -0.72 \\
\hline $\begin{array}{l}\text { Diabla Gora, } \\
\text { PL0005R }\end{array}$ & 68 & 0.20 & 1.28 & 3.39 & -0.62 & 56 & 0.06 & 1.86 & 3.77 & -0.51 \\
\hline $\begin{array}{l}\text { Ähtäri, } \\
\text { FI0004R }\end{array}$ & 12 & 0.07 & 0.68 & 2.59 & -0.74 & 9 & 0.58 & 0.81 & 2.82 & -0.71 \\
\hline $\begin{array}{l}\text { Virolahti II } \\
\text { FI0017R }\end{array}$ & 18 & -0.07 & 0.70 & 4.95 & -0.86 & 13 & -0.03 & 1.02 & 4.68 & -0.78 \\
\hline $\begin{array}{l}\text { Hailuoto II, } \\
\text { FI0053R }\end{array}$ & 13 & -0.18 & 0.60 & 3.50 & -0.83 & 4 & - & - & - & - \\
\hline $\begin{array}{l}\text { Lahemaa, } \\
\text { EE0009R }\end{array}$ & 47 & 0.46 & 1.09 & 1.43 & -0.24 & 36 & 0.29 & 1.12 & 1.76 & -0.37 \\
\hline $\begin{array}{l}\text { Preila } \\
\text { LT0015R }\end{array}$ & 53 & 0.48 & 1.23 & 2.26 & -0.45 & 40 & 0.45 & 2.04 & 2.53 & -0.19 \\
\hline
\end{tabular}


Table S5: Seasonal averages of wet deposition of nitrate for stations of the EMEP monitoring network in the Baltic Sea region. CMAQ model results were taken from the CD04 grid domains. For spring (MAM), summer (JJA) and autumn (SON), the mean value of model ( $\left.\mu_{\text {Mod }}\right)$ and mean value of observations ( $\mu_{0 b s}$ ) is given based on daily sums. Unit of mean values is $\operatorname{mg}(\mathrm{N})$ $\mathrm{m}^{-2} \mathrm{~d}^{-1}$. Observational and model data was evaluated independently. $N_{\text {Obs }}$ is the number of observations in the respective season, used to calculate $\mu_{\mathrm{obs}} . N_{\text {Mod }}$ is the number of days with simulated precipitation in the respective season, used to calculate $\mu_{\text {Mod. }}$.

\begin{tabular}{|c|c|c|c|c|c|c|c|c|c|c|c|c|}
\hline \multirow{2}{*}{$\begin{array}{l}\text { Station, } \\
\text { Code }\end{array}$} & \multicolumn{4}{|c|}{ Spring } & \multicolumn{4}{|c|}{ Summer } & \multicolumn{4}{|c|}{ Winter } \\
\hline & $N_{\text {Mod }}$ & $N_{\text {obs }}$ & $\mu_{\mathrm{Mod}}$ & $\mu \mathrm{obs}$ & $N_{\text {Mod }}$ & $N_{\mathrm{obs}}$ & $\mu_{\mathrm{Mod}}$ & $\mu_{\mathrm{Obs}}$ & $N_{\text {Mod }}$ & Nobs & $\mu_{\text {Mod }}$ & $\mu \mathrm{obs}$ \\
\hline $\begin{array}{l}\text { Zingst, } \\
\text { DE0009R }\end{array}$ & 9 & 11 & 1.71 & 3.54 & 18 & 10 & 2.25 & 5.71 & 22 & 13 & 1.85 & 4.98 \\
\hline $\begin{array}{l}\text { Råö } \\
\text { SE0014R }\end{array}$ & 13 & 24 & 3.38 & 3.48 & 15 & 29 & 1.51 & 3.80 & 42 & 48 & 2.23 & 2.83 \\
\hline $\begin{array}{l}\text { Leba, } \\
\text { PL0004R }\end{array}$ & 14 & 24 & 1.76 & 1.47 & 10 & 31 & 2.88 & 5.27 & 33 & 58 & 1.43 & 2.21 \\
\hline $\begin{array}{l}\text { Diabla Gora, } \\
\text { PL0005R }\end{array}$ & 20 & 29 & 1.75 & 3.64 & 19 & 25 & 2.22 & 4.06 & 30 & 46 & 1.60 & 3.06 \\
\hline $\begin{array}{l}\text { Ähtäri, } \\
\text { FI0004R }\end{array}$ & 7 & 9 & 1.09 & 3.25 & 14 & 9 & 0.92 & 2.73 & 17 & 12 & 1.36 & 3.46 \\
\hline $\begin{array}{l}\text { Virolahti II } \\
\text { FI0017R }\end{array}$ & 13 & 11 & 1.98 & 3.30 & 14 & 9 & 1.33 & 3.48 & 36 & 16 & 1.26 & 6.50 \\
\hline $\begin{array}{l}\text { Hailuoto II, } \\
\text { FI0053R }\end{array}$ & 9 & 11 & 0.70 & 3.16 & 11 & 8 & 0.86 & 1.61 & 23 & 15 & 1.03 & 3.24 \\
\hline $\begin{array}{l}\text { Lahemaa, } \\
\text { EE0009R }\end{array}$ & 17 & 29 & 1.37 & 1.64 & 17 & 21 & 1.24 & 1.27 & 24 & 39 & 1.32 & 2.11 \\
\hline $\begin{array}{l}\text { Preila } \\
\text { LT0015R }\end{array}$ & 14 & 10 & 1.52 & 3.09 & 17 & 24 & 2.63 & 2.16 & 29 & 41 & 1.61 & 3.04 \\
\hline
\end{tabular}


Table S6: Seasonal averages of wet deposition of ammonium for stations of the EMEP monitoring network in the Baltic Sea region. CMAQ model results were taken from the CD04 grid domains. For spring (MAM), summer (JJA) and autumn (SON), the mean value of model ( $\mu$ Mod) and mean value of observations ( $\mu$ obs) is given based on daily sums. Unit of mean values is $\mathrm{mg}(\mathrm{N}) \mathrm{m}^{-2} \mathrm{~d}^{-1}$. Observational and model data was evaluated independently. $N_{\text {Obs }}$ is the number of observations in the respective season, used to calculate $\mu_{0 \text { bs. }} N_{\text {Mod }}$ is the number of days with simulated precipitation in the respective season, used to calculate $\mu_{\text {Mod. }}$.

\begin{tabular}{|c|c|c|c|c|c|c|c|c|c|c|c|c|}
\hline \multirow{2}{*}{$\begin{array}{l}\text { Station, } \\
\text { Code }\end{array}$} & \multicolumn{4}{|c|}{ Spring } & \multicolumn{4}{|c|}{ Summer } & \multicolumn{4}{|c|}{ Winter } \\
\hline & $N_{\text {Mod }}$ & $N_{\mathrm{Obs}}$ & $\mu_{\mathrm{Mod}}$ & $\mu_{\mathrm{obs}}$ & $N_{\text {Mod }}$ & $N_{\mathrm{Obs}}$ & $\mu_{\mathrm{Mod}}$ & $\mu_{\mathrm{obs}}$ & $N_{\text {Mod }}$ & $N_{\text {Obs }}$ & $\mu_{\text {Mod }}$ & $\mu_{\mathrm{obs}}$ \\
\hline $\begin{array}{l}\text { Zingst, } \\
\text { DE0009R }\end{array}$ & 9 & 11 & 2.36 & 7.73 & 19 & 11 & 2.01 & 9.68 & 19 & 14 & 2.30 & 6.33 \\
\hline $\begin{array}{l}\text { Råö } \\
\text { SE0014R }\end{array}$ & 13 & 24 & 3.87 & 5.03 & 17 & 11 & 1.02 & 1.64 & 34 & 40 & 2.17 & 2.42 \\
\hline $\begin{array}{l}\text { Leba, } \\
\text { PL0004R }\end{array}$ & 13 & 22 & 2.12 & 2.20 & 14 & 7 & 1.08 & 1.94 & 28 & 55 & 1.33 & 2.21 \\
\hline $\begin{array}{l}\text { Diabla Gora, } \\
\text { PL0005R }\end{array}$ & 23 & 27 & 2.32 & 6.18 & 14 & 9 & 1.44 & 3.51 & 28 & 39 & 1.47 & 2.44 \\
\hline $\begin{array}{l}\text { Ähtäri, } \\
\text { FI0004R }\end{array}$ & 7 & 9 & 1.11 & 3.03 & 14 & 7 & 1.08 & 1.94 & 17 & 11 & 0.98 & 2.28 \\
\hline $\begin{array}{l}\text { Virolahti II } \\
\text { FI0017R }\end{array}$ & 14 & 11 & 2.61 & 2.98 & 14 & 9 & 1.44 & 3.51 & 28 & 15 & 1.12 & 5.62 \\
\hline $\begin{array}{l}\text { Hailuoto II, } \\
\text { FI0053R }\end{array}$ & 9 & 11 & 0.75 & 3.86 & 10 & 8 & 1.00 & 1.51 & 18 & 14 & 0.74 & 3.64 \\
\hline $\begin{array}{l}\text { Lahemaa, } \\
\text { EE0009R }\end{array}$ & 17 & 22 & 1.64 & 2.01 & 17 & 11 & 1.02 & 1.64 & 23 & 17 & 0.90 & 1.78 \\
\hline $\begin{array}{l}\text { Preila } \\
\text { LT0015R }\end{array}$ & 15 & 10 & 1.60 & 4.19 & 16 & 22 & 3.24 & 2.57 & 23 & 39 & 1.25 & 2.42 \\
\hline
\end{tabular}


Table S7: Statistical evaluation of modelled $\mathrm{O}_{3}$ concentrations (in ppbv) with measurements of the EMEP monitoring network in the Baltic Sea region based on daily means for the entire year and for summer (JJA). Statistical indicators: mean values of model ( $\left.\mu_{\text {Mod }}\right)$, mean values of observations ( $\left.\mu_{0 \mathrm{bs}}\right)$, Spearman's correlation coefficient $\left(\mathrm{R}_{\mathrm{Spr}}\right)$, normalized mean bias (NMB) and root mean square error of the modelled values (RMSE; in ppbv). All stations of the EMEP network located within the CD04 grid domain with available measurements were considered. $N$ is the number of the available daily mean measurements at the respective station.

\begin{tabular}{|c|c|c|c|c|c|c|c|c|c|c|c|c|}
\hline \multirow{2}{*}{$\begin{array}{l}\text { Station } \\
\text { Code }\end{array}$} & \multicolumn{6}{|c|}{ CD04 annual } & \multicolumn{6}{|c|}{ CD04 summer } \\
\hline & $N$ & $\mathrm{R}_{\mathrm{Spr}}$ & $\mu_{\mathrm{Mod}}$ & $\mu \mathrm{obs}$ & NMB & RMSE & $N$ & $\mathrm{R}_{\mathrm{Spr}}$ & $\mu_{\mathrm{Mod}}$ & $\mu_{\mathrm{obs}}$ & NMB & RMSE \\
\hline $\begin{array}{l}\text { Zingst, } \\
\text { DE0009R }\end{array}$ & 366 & 0.80 & 27.3 & 27.8 & -0.02 & 5.9 & 92 & 0.64 & 29.0 & 33.0 & -0.12 & 6.5 \\
\hline $\begin{array}{l}\text { Keldsnor, } \\
\text { DK0005R }\end{array}$ & 357 & 0.66 & 26.3 & 28.1 & -0.07 & 7.2 & 86 & 0.29 & 26.8 & 32.9 & -0.18 & 9.7 \\
\hline $\begin{array}{l}\text { Risoe, } \\
\text { DK0012R }\end{array}$ & 366 & 0.74 & 26.1 & 29.3 & -0.11 & 6.1 & 92 & 0.48 & 26.9 & 35.0 & -0.23 & 10.0 \\
\hline $\begin{array}{l}\text { Ähtäri II, } \\
\text { FI0037R }\end{array}$ & 358 & 0.79 & 23.8 & 24.8 & -0.04 & 5.4 & 92 & 0.78 & 23.6 & 22.3 & 0.06 & 4.4 \\
\hline $\begin{array}{l}\text { Virolahti II } \\
\text { FI0017R }\end{array}$ & 358 & 0.70 & 25.0 & 26.6 & -0.06 & 7.0 & 91 & 0.77 & 26.1 & 24.5 & 0.07 & 5.5 \\
\hline $\begin{array}{l}\text { Utö, } \\
\text { FI0009R }\end{array}$ & 362 & 0.74 & 28.1 & 31.1 & -0.09 & 6.4 & 90 & 0.68 & 28.9 & 34.6 & -0.17 & 7.6 \\
\hline $\begin{array}{l}\text { Rucava, } \\
\text { LV0010R }\end{array}$ & 347 & 0.73 & 27.8 & 33.1 & -0.16 & 9.5 & 92 & 0.67 & 30.5 & 32.3 & -0.06 & 6.7 \\
\hline $\begin{array}{l}\text { Vilsandi, } \\
\text { EE0011R }\end{array}$ & 361 & 0.80 & 28.8 & 31.7 & -0.09 & 6.1 & 90 & 0.83 & 31.1 & 35.7 & -0.13 & 6.3 \\
\hline $\begin{array}{l}\text { Lahemaa, } \\
\text { EE0009R }\end{array}$ & 365 & 0.69 & 25.4 & 26.5 & -0.04 & 6.7 & 91 & 0.69 & 26.9 & 26.4 & 0.02 & 5.3 \\
\hline $\begin{array}{l}\text { Preila } \\
\text { LT0015R }\end{array}$ & 364 & 0.77 & 29.2 & 29.8 & -0.02 & 6.7 & 91 & 0.48 & 33.2 & 33.5 & -0.01 & 7.2 \\
\hline
\end{tabular}


Table S8: Statistical evaluation of modelled $\mathrm{NO}_{2}$ concentrations (in ppbv) with measurements of the EMEP monitoring network in the Baltic Sea region based on daily means for the entire year and for summer (JJA). Statistical indicators: mean values of model ( $\left.\mu_{\text {Mod }}\right)$, mean values of observations ( $\left.\mu_{\mathrm{Obs}}\right)$, Spearman's correlation coefficient $\left(\mathrm{R}_{\mathrm{Spr}}\right)$, normalized mean bias (NMB) and root mean square error of the modelled values (RMSE; in ppbv). All stations of the EMEP network located within the CD04 grid domain with available measurements were considered. $N$ is the number of the available daily mean measurements at the respective station.

\begin{tabular}{|c|c|c|c|c|c|c|c|c|c|c|c|c|}
\hline \multirow{2}{*}{$\begin{array}{l}\text { Station } \\
\text { Code }\end{array}$} & \multicolumn{6}{|c|}{ CD04 annual } & \multicolumn{6}{|c|}{ CD04 summer } \\
\hline & $N$ & $\mathrm{R}_{\mathrm{Spr}}$ & $\mu$ Mod & $\mu_{\mathrm{obs}}$ & NMB & RMSE & $N$ & $\mathrm{R}_{\mathrm{Spr}}$ & $\mu \mathrm{Mod}$ & $\mu_{\mathrm{obs}}$ & NMB & RMSE \\
\hline $\begin{array}{l}\text { Zingst, } \\
\text { DE0009R }\end{array}$ & 355 & 0.73 & 3.8 & 3.4 & 0.10 & 2.04 & 88 & 0.58 & 2.9 & 2.4 & 0.22 & 1.50 \\
\hline $\begin{array}{l}\text { Keldsnor, } \\
\text { DK0005R }\end{array}$ & 331 & 0.84 & 5.1 & 4.0 & 0.27 & 3.24 & 86 & 0.82 & 4.9 & 3.2 & 0.52 & 3.46 \\
\hline $\begin{array}{l}\text { Anholt, } \\
\text { DK0008R }\end{array}$ & 283 & 0.81 & 3.7 & 2.6 & 0.42 & 2.67 & 92 & 0.80 & 3.6 & 2.0 & 0.83 & 2.91 \\
\hline $\begin{array}{l}\text { Risoe, } \\
\text { DK0012R }\end{array}$ & 351 & 0.83 & 4.7 & 4.6 & 0.02 & 1.97 & 88 & 0.83 & 3.5 & 2.9 & 0.19 & 1.19 \\
\hline $\begin{array}{l}\text { Ähtäri II, } \\
\text { FI0037R }\end{array}$ & 349 & 0.86 & 1.0 & 1.2 & -0.22 & 1.05 & 78 & 0.47 & 0.3 & 0.4 & -0.31 & 0.18 \\
\hline $\begin{array}{l}\text { Virolahti II } \\
\text { FI0017R }\end{array}$ & 365 & 0.65 & 2.1 & 2.6 & -0.18 & 2.60 & 92 & 0.54 & 1.5 & 1.5 & -0.01 & 0.90 \\
\hline $\begin{array}{l}\text { Hyytiälä, } \\
\text { FI0050R }\end{array}$ & 142 & 0.90 & 1.3 & 1.8 & -0.28 & 1.63 & 32 & 0.70 & 0.3 & 0.4 & -0.10 & 0.19 \\
\hline $\begin{array}{l}\text { Utö, } \\
\text { FI0009R }\end{array}$ & 331 & 0.71 & 1.9 & 1.6 & 0.18 & 1.26 & 87 & 0.66 & 2.1 & 1.4 & 0.48 & 1.50 \\
\hline $\begin{array}{l}\text { Rucava, } \\
\text { LV0010R }\end{array}$ & 357 & 0.74 & 1.9 & 1.3 & 0.44 & 1.35 & 83 & 0.30 & 1.0 & 0.7 & 0.36 & 0.49 \\
\hline $\begin{array}{l}\text { Vilsandi, } \\
\text { EE0011R }\end{array}$ & 340 & 0.69 & 1.6 & 1.3 & 0.24 & 1.12 & 89 & 0.49 & 1.4 & 0.8 & 0.71 & 1.44 \\
\hline $\begin{array}{l}\text { Lahemaa, } \\
\text { EE0009R }\end{array}$ & 335 & 0.76 & 1.8 & 1.5 & 0.20 & 1.21 & 84 & 0.71 & 1.2 & 0.8 & 0.59 & 0.75 \\
\hline $\begin{array}{l}\text { Preila } \\
\text { LT0015R }\end{array}$ & 333 & 0.65 & 1.8 & 1.8 & 0.04 & 1.54 & 89 & 0.42 & 1.0 & 1.2 & -0.18 & 0.59 \\
\hline
\end{tabular}


Table S9: Statistical evaluation of modelled $\mathrm{SO}_{2}$ concentrations (in ppbv) with measurements of the EMEP monitoring network in the Baltic Sea region based on daily means for the entire year and for summer (JJA). Statistical indicators: include mean values of model ( $\left.\mu_{\mathrm{Mod}}\right)$, mean values of observations ( $\left.\mu_{0 \mathrm{obs}}\right)$, Spearman's correlation coefficient $\left(\mathrm{R}_{\mathrm{Spr}}\right)$, normalized mean bias (NMB) and root mean square error of the modelled values (RMSE; in ppbv). All stations of the EMEP network located within the CD04 grid domain with available measurements were considered. $N$ is the number of the available daily mean measurements at the respective station.

\begin{tabular}{|c|c|c|c|c|c|c|c|c|c|c|c|c|}
\hline \multirow{2}{*}{$\begin{array}{l}\text { Station } \\
\text { Code }\end{array}$} & \multicolumn{6}{|c|}{ CD04 annual } & \multicolumn{6}{|c|}{ CD04 summer } \\
\hline & $N$ & $R_{\mathrm{Spr}}$ & $\mu_{\mathrm{Mo}}$ & $\mu_{\mathrm{Obs}}$ & NMB & $\mathrm{RM}$ & $N$ & $R_{\mathrm{Spr}}$ & $\mu_{\mathrm{Mod}}$ & $\mu \mathrm{obs}$ & NMB & RMS \\
\hline $\begin{array}{l}\text { Zingst, } \\
\text { DE0009R }\end{array}$ & 366 & 0.54 & 1.21 & 0.87 & 0.40 & 0.98 & 92 & 0.54 & 0.75 & 0.80 & -0.07 & 0.37 \\
\hline $\begin{array}{l}\text { Anholt, } \\
\text { DK0008R }\end{array}$ & 351 & 0.73 & 0.87 & 0.37 & 1.33 & 0.79 & 92 & 0.73 & 0.76 & 0.43 & 0.76 & 0.62 \\
\hline $\begin{array}{l}\text { Risoe, } \\
\text { DK0012R }\end{array}$ & 350 & 0.70 & 1.03 & 0.42 & 1.46 & 0.91 & 92 & 0.73 & 0.68 & 0.37 & 0.81 & 0.45 \\
\hline $\begin{array}{l}\text { Ähtäri II, } \\
\text { FI0037R }\end{array}$ & 50 & 0.61 & 0.50 & 0.35 & 1.15 & 0.40 & 12 & 0.04 & 0.19 & 0.07 & 1.84 & 0.17 \\
\hline $\begin{array}{l}\text { Virolahti II } \\
\text { FI0017R }\end{array}$ & 282 & 0.72 & 1.24 & 1.26 & -0.02 & 1.22 & 50 & 0.47 & 0.74 & 0.43 & 0.73 & 0.55 \\
\hline $\begin{array}{l}\text { Utö, } \\
\text { FI0009R }\end{array}$ & 318 & 0.74 & 0.78 & 0.65 & 0.20 & 0.57 & 75 & 0.60 & 0.59 & 0.50 & 0.18 & 0.41 \\
\hline $\begin{array}{l}\text { Rucava, } \\
\text { LV0010R }\end{array}$ & 359 & 0.58 & 1.25 & 0.48 & 1.62 & 1.50 & 92 & 0.29 & 0.60 & 0.35 & 0.71 & 0.52 \\
\hline $\begin{array}{l}\text { Vilsandi, } \\
\text { EE0011R }\end{array}$ & 283 & 0.69 & 0.84 & 0.58 & 0.44 & 0.78 & 67 & 0.39 & 0.48 & 0.39 & 0.24 & 0.25 \\
\hline $\begin{array}{l}\text { Lahemaa, } \\
\text { EE0009R }\end{array}$ & 270 & 0.57 & 1.13 & 1.17 & -0.04 & 1.45 & 75 & 0.42 & 0.61 & 0.58 & 0.05 & 0.41 \\
\hline $\begin{array}{l}\text { Preila } \\
\text { LT0015R }\end{array}$ & 302 & 0.57 & 1.29 & 0.60 & 1.15 & 1.47 & 92 & 0.30 & 0.55 & 0.24 & 1.28 & 0.43 \\
\hline
\end{tabular}




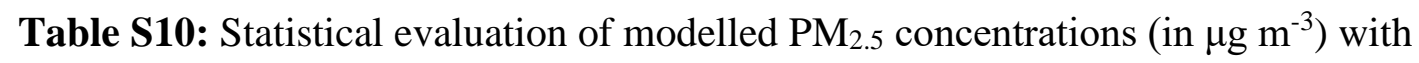
measurements of the EMEP monitoring network in the Baltic Sea region based on daily means for the entire year and for summer (JJA). Statistical indicators: include mean values of model ( $\left.\mu_{\text {Mod }}\right)$, mean values of observations ( $\left.\mu_{\mathrm{Obs}}\right)$, Spearman's correlation coefficient $\left(\mathrm{R}_{\mathrm{Spr}}\right)$, normalized mean bias (NMB) and root mean square error of the modelled values (RMSE; in $\mu \mathrm{g} \mathrm{m}^{-3}$ ). All stations of the EMEP network located within the CD04 grid domain with available measurements were considered. $N$ is the number of the available daily mean measurements at the respective station.

\begin{tabular}{l||cccccc|cccccc}
\hline \hline $\begin{array}{l}\text { Station } \\
\text { Code }\end{array}$ & \multicolumn{6}{|c|}{ CD04 annual } & \multicolumn{6}{c}{ CD04 summer } \\
\cline { 2 - 11 } & $N$ & $\mathrm{R}_{\text {Spr }}$ & $\mu_{\text {Mod }}$ & $\mu_{\text {obs }}$ & NMB & RMSE & $N$ & $\mathrm{R}_{\text {Spr }}$ & $\mu_{\text {Mod }}$ & $\mu_{\text {obs }}$ & NMB & RMSE \\
\hline \hline $\begin{array}{l}\text { Råö } \\
\text { SE0014R }\end{array}$ & 351 & 0.49 & 4.9 & 5.6 & -0.13 & 4.4 & 87 & 0.36 & 2.5 & 4.9 & -0.50 & 3.5 \\
\hline $\begin{array}{l}\text { Diabla Gora, } \\
\text { PL0005R }\end{array}$ & 365 & 0.82 & 7.8 & 13.8 & -0.44 & 8.5 & 92 & 0.70 & 2.5 & 8.7 & -0.71 & 6.8 \\
\hline $\begin{array}{l}\text { Vavihill, } \\
\text { SE0011R }\end{array}$ & 252 & 0.55 & 6.3 & 8.2 & -0.23 & 5.2 & 84 & 0.62 & 2.8 & 6.5 & -0.58 & 4.5 \\
\hline $\begin{array}{l}\text { Aspvreten, } \\
\text { SE0012R }\end{array}$ & 254 & 0.51 & 4.9 & 6.5 & -0.24 & 4.3 & 78 & 0.61 & 1.9 & 6.0 & -0.68 & 4.7 \\
\hline $\begin{array}{l}\text { Utö, } \\
\text { FI0009R }\end{array}$ & 357 & 0.63 & 3.9 & 5.2 & -0.26 & 3.4 & 90 & 0.71 & 1.8 & 5.2 & -0.65 & 3.8 \\
\hline $\begin{array}{l}\text { Rucava, } \\
\text { LV0010R }\end{array}$ & 327 & 0.29 & 6.8 & 10.8 & -0.37 & 10.4 & 78 & -0.19 & 2.5 & 7.5 & -0.66 & 6.5 \\
\hline $\begin{array}{l}\text { Vilsandi, } \\
\text { EE0011R }\end{array}$ & 342 & 0.67 & 4.6 & 5.5 & -0.16 & 3.9 & 84 & 0.73 & 2.0 & 4.1 & -0.52 & 3.0 \\
\hline $\begin{array}{l}\text { Lahemaa, } \\
\text { EE0009R }\end{array}$ & 343 & 0.60 & 6.0 & 5.5 & 0.09 & 4.6 & 87 & 0.32 & 1.8 & 3.6 & -0.50 & 2.7 \\
\hline \hline
\end{tabular}


COSMO-CLM and Observations: Monthly Summer Precipitation 2012
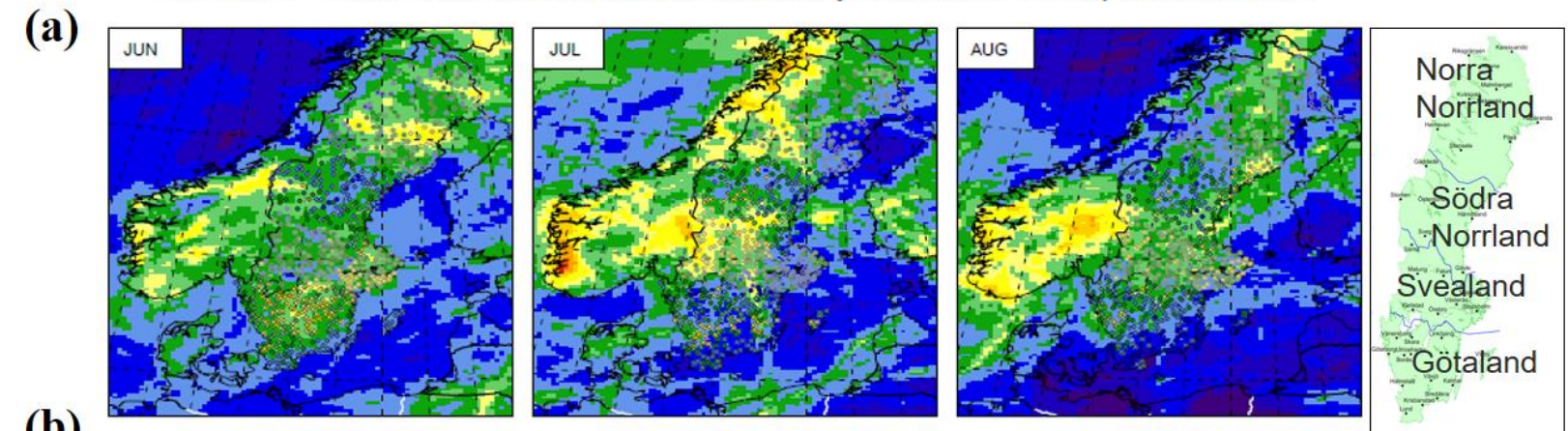

(b)
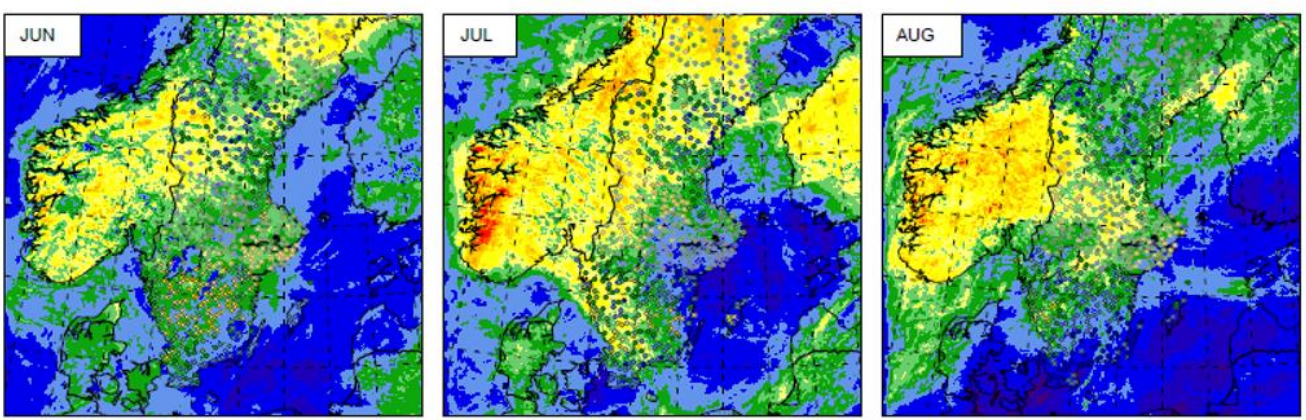

(c)
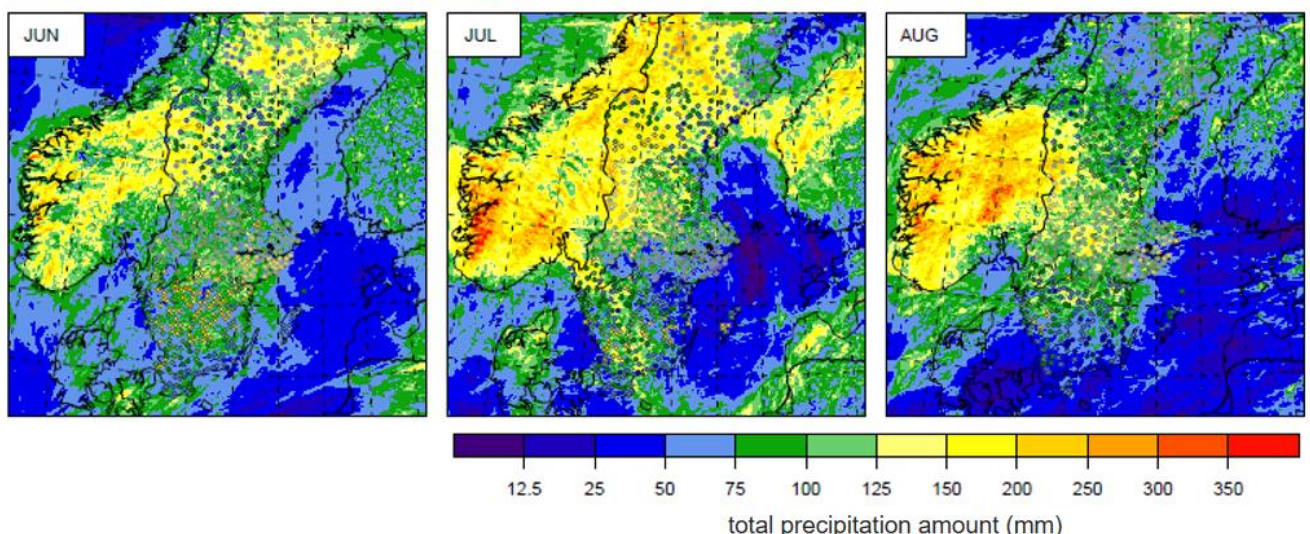

Figure S1. Monthly accumulated precipitation (mm) maps for summer months (JJA) in 2012: computed precipitation fields from different configurations of COSMO-CLM compared to observations at meteorological stations shown as circles filled with corresponding colour of observed precipitation: (a) COSMO-CLM on 0.11 degrees (“011”), (b) COSMO-CLM on 0.025 degrees with parameterised convection ("0025_Tiedtke") and (c) COSMO-CLM on 0.025 degrees with convection-permitting configuration (“0025_convper"), as used for the CMAQ high resolution domain. Observation data is based on precipitation measurements at 1804 stations of the Swedish meteorological network from SMHI. Inset in the top right corner shows the definition of the four regions of Sweden with blue border lines. 
Probability distribution of differences in total precipitation SMHI station - CCLM 2012
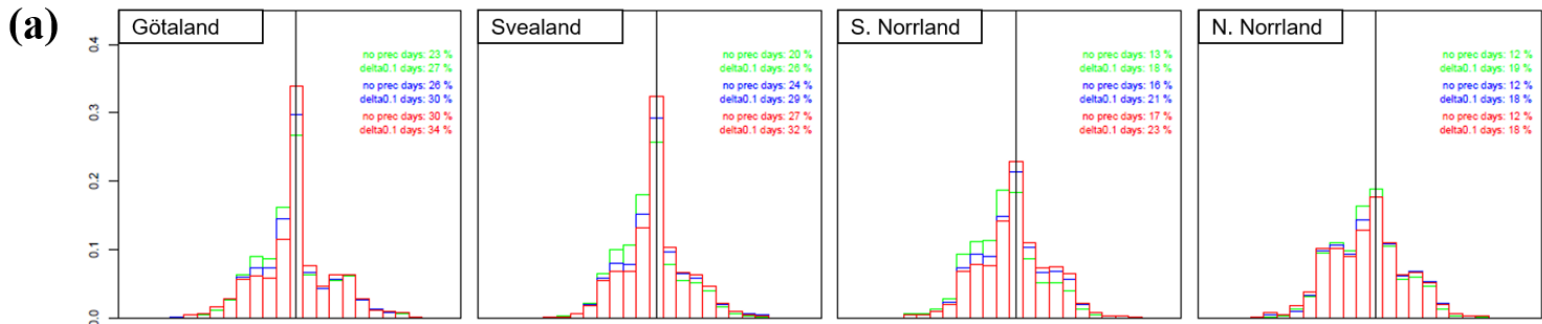

(b)
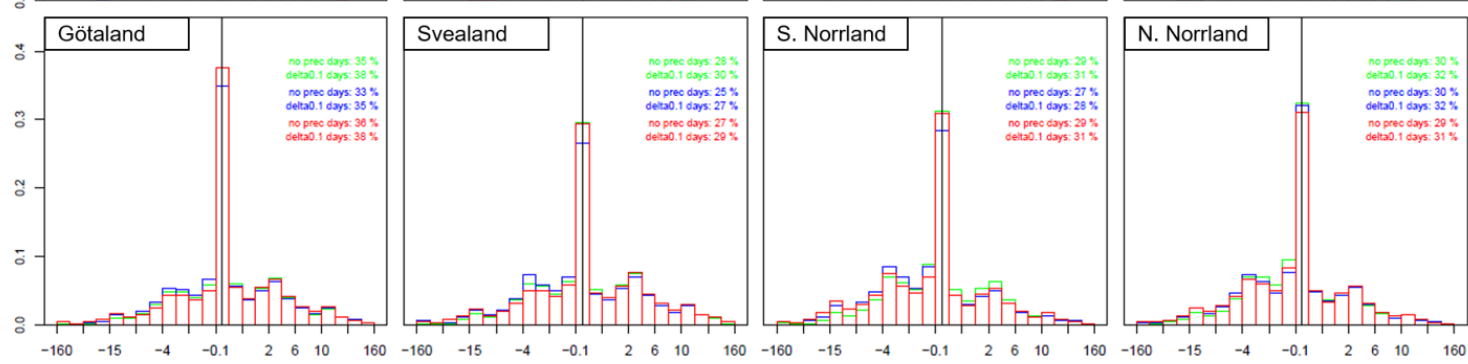

무일 011 - 011 0025_Tiedtke

Figure S2. Probability distribution of the differences in daily precipitation sums $\left(\mathrm{mm} \mathrm{d}^{-1}\right)$ between the SMHI station observations and COSMO-CLM with different configurations ("011", "0025_Tiedtke", and "0025_convper") in the four regions of Sweden (from left to right: Götaland, Svealand, Södra Norrland, Norra Norland): (a) for the winter months (JFD) of 2012 and (b) for the summer months (JJA) of 2012. The percentage fraction of days with zero difference between model and observation ("no prec." days) and the percentage fraction of days with difference of $\pm 0.1 \mathrm{~mm} \mathrm{~d}^{-1}$ ("delta0.1" days) is indicated in the plots for each model configuration. 
Probability distribution of differences in total precipitation SMHI station - CCLM Götaland 2012
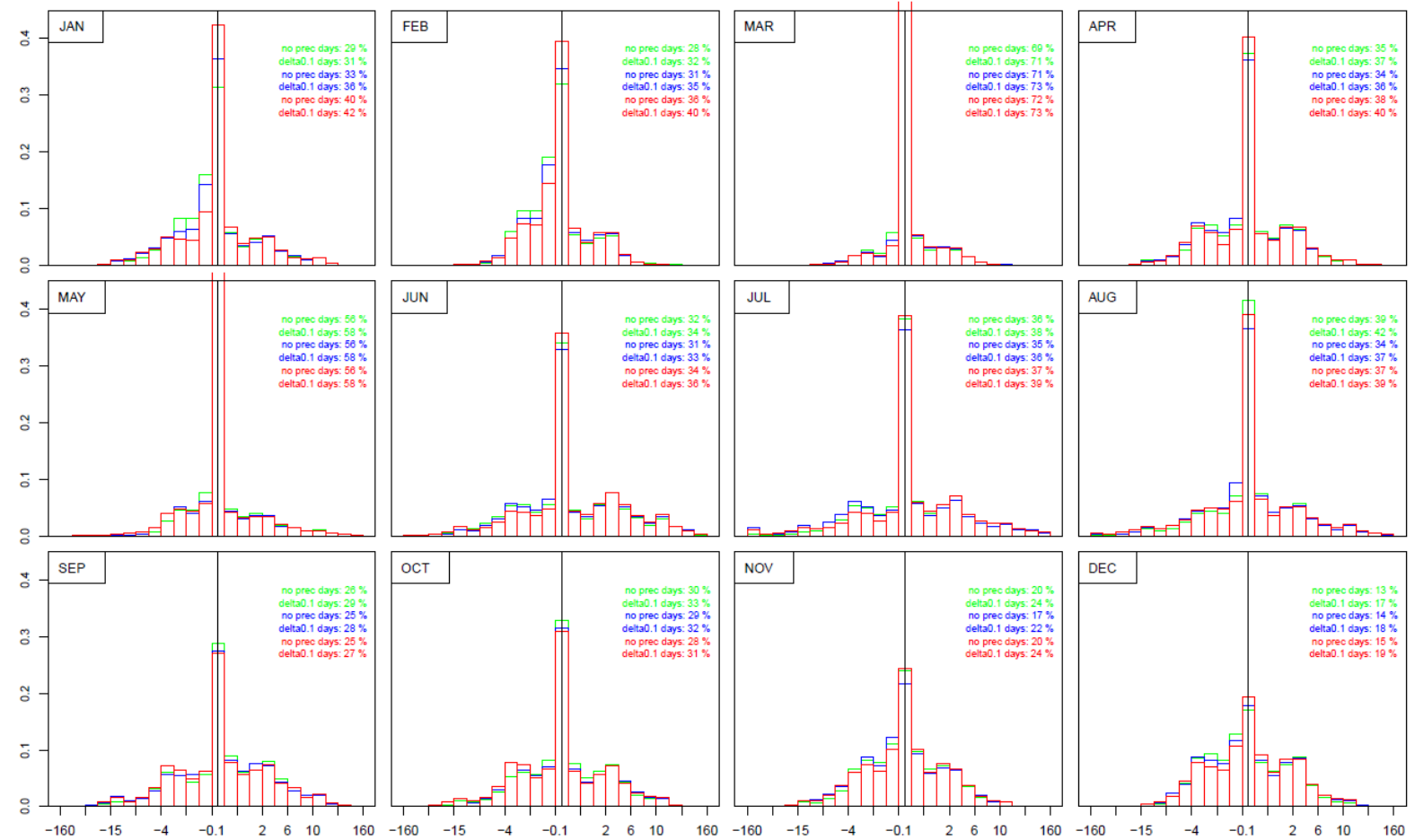

$\begin{array}{ll}0 & 011 \\ \text { 0. } 0025 \text { Tiedtke } \\ \text { 0025_convper }\end{array}$

Figure S3. Probability distribution of the differences in daily precipitation sums $\left(\mathrm{mm} \mathrm{d}^{-1}\right)$ between the SMHI station observations and COSMO-CLM with different configurations (“011”, “0025_Tiedtke”, and "0025_convper") in Götaland for the months of 2012. The percentage fraction of days with zero difference between model and observation ("no prec. days") and the percentage fraction of days with difference of $\pm 0.1 \mathrm{~mm} \mathrm{~d}^{-1}$ ("delta0.1 days") is indicated in the plots for each model configuration 
(a) Anholt, DK0008R

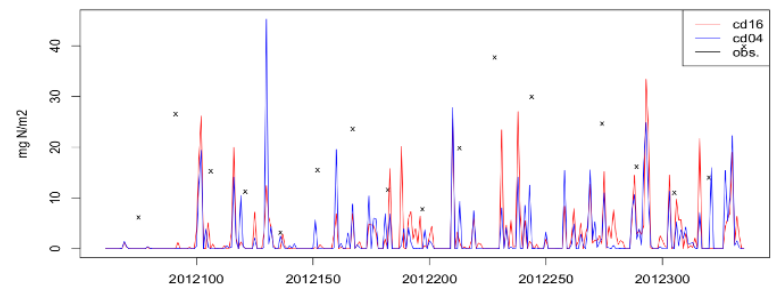

(c) Diabla Gora, PL0005R

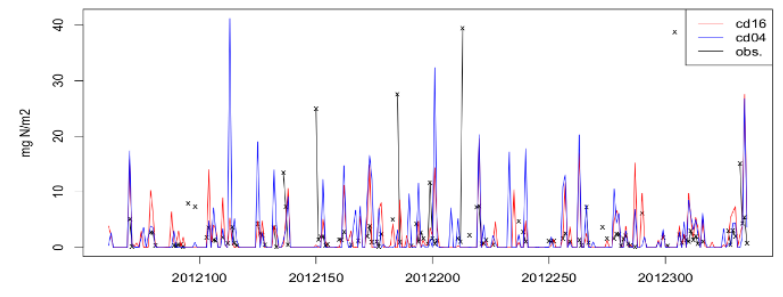

(e) Keldsnor, DK0005R

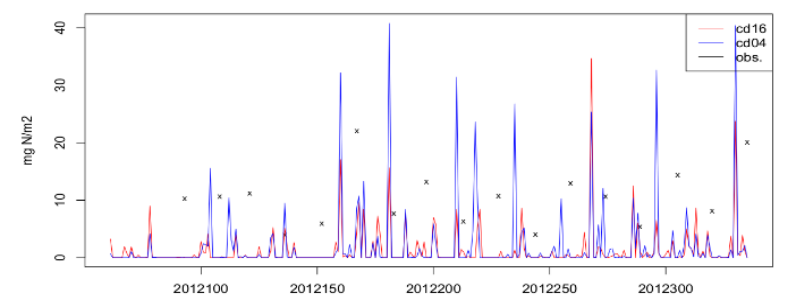

(g) Hailuoto II, FI0053R

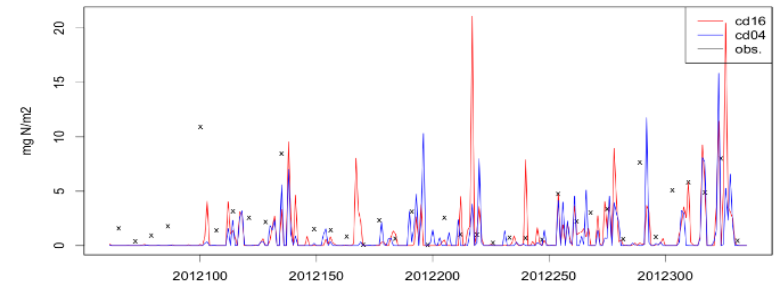

(i) Vilsandi, EE0011R

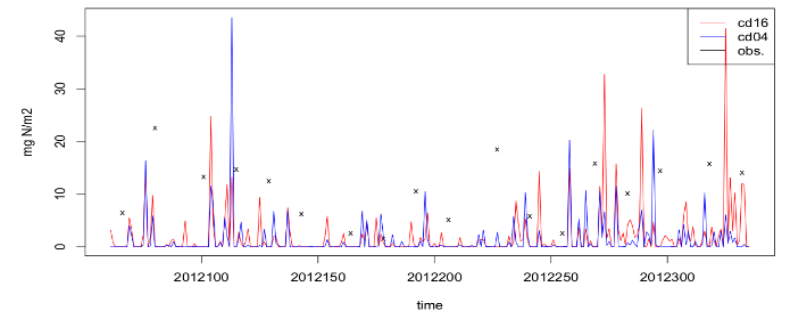

(b) Vavihill, SE0011R

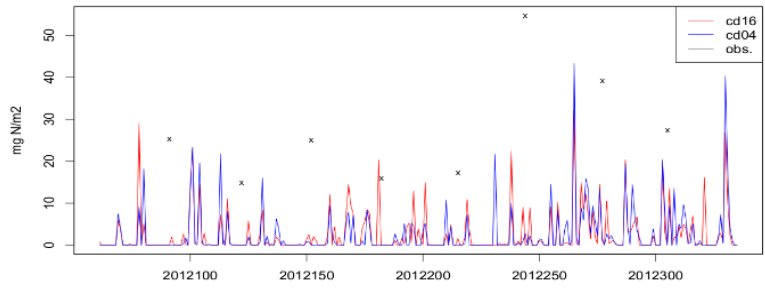

(d) Aspvreten, SE0012R

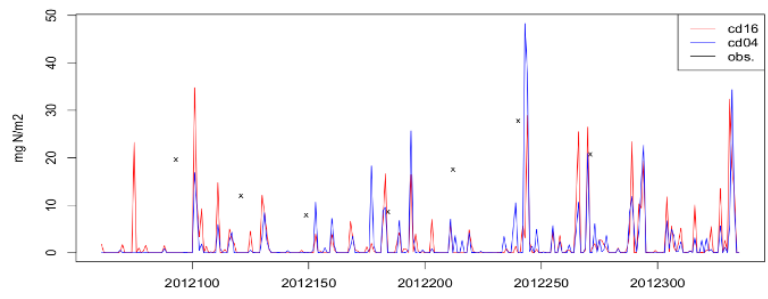

(f) Lahemaa, EE0009R

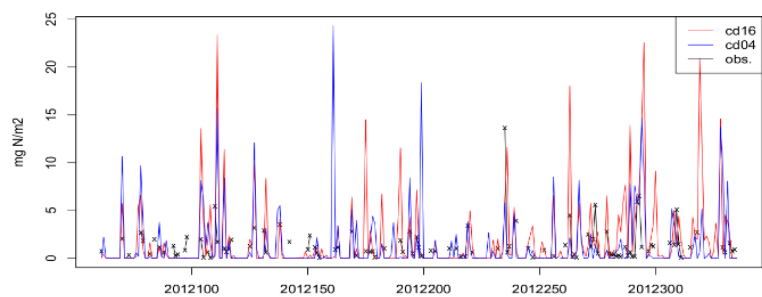

(h) Rucava, LV0010R

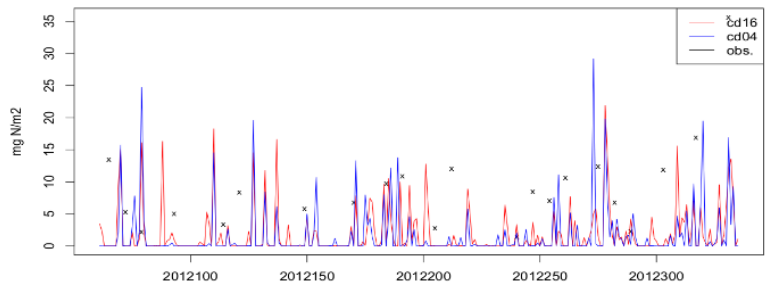

(j) Sepstrup Sande, DK0022R

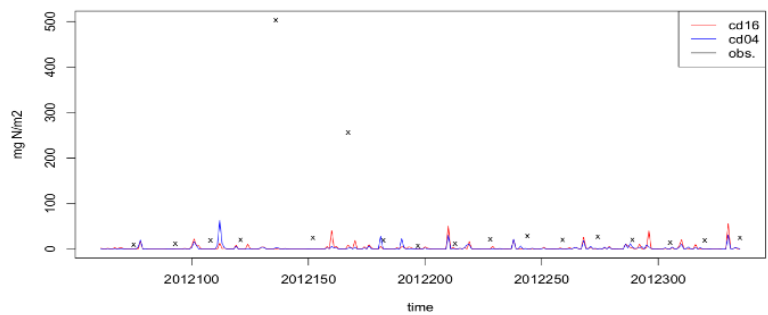

Figure S4. Comparison of modelled wet deposition of nitrate as daily sums $\left(\mathrm{mg}(\mathrm{N}) \mathrm{m}^{-2} \mathrm{~d}^{-1}\right)$ from the 16-km resolution grid (red) and 4km-resolution grid (blue) against observed daily sums of nitrate wet deposition (black crosses) at regional background stations around the Baltic Sea from the EMEP monitoring network: (a) Anholt, DK0008R, (b) Vavihill, SE0011R, (c) Diabla Gora, PL0005R, (d) Aspvreten, SE0012R, (e) Keldsnor, DK0005R, (f) Lahemaa, EE0009R, (g) Hailuoto II, FI0053R, (h) Rucava, LV0010R, (i) Vilsandi, EE0011R, and (j) Sepstrup Sande, DK0022R. Comparison time period: 1 March to 30 November 2012. 
(a) Zingst, DE0009R

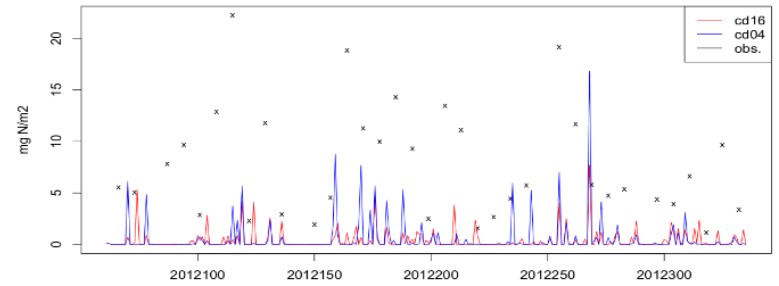

(c) Diabla Gora, PL0005R

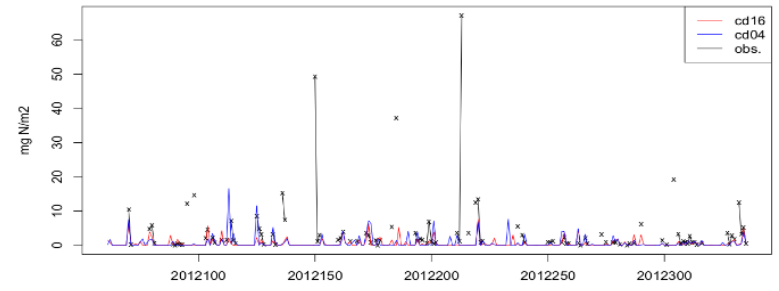

(e) Ahtari, F10004R

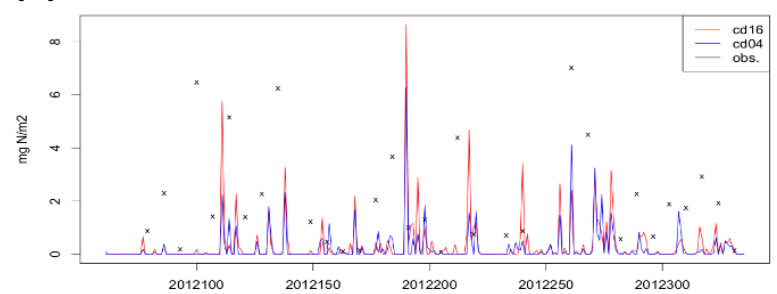

(g) Hailuoto II, FI0053R

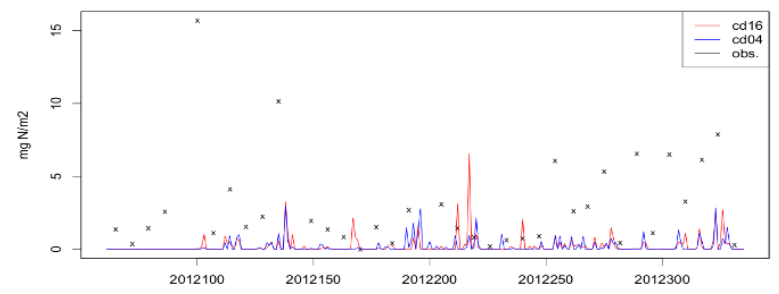

(i) Vilsandi, EE0011R

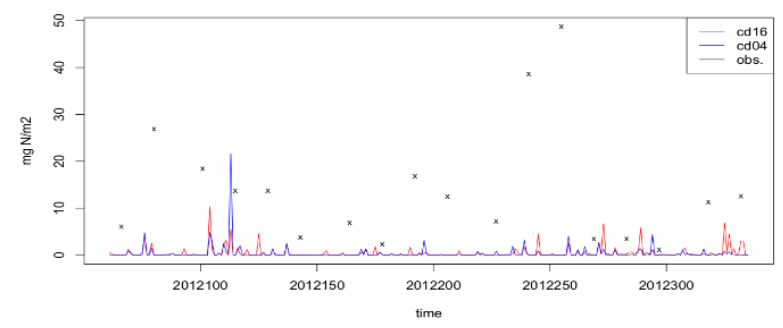

(b) Råö, SE0014R

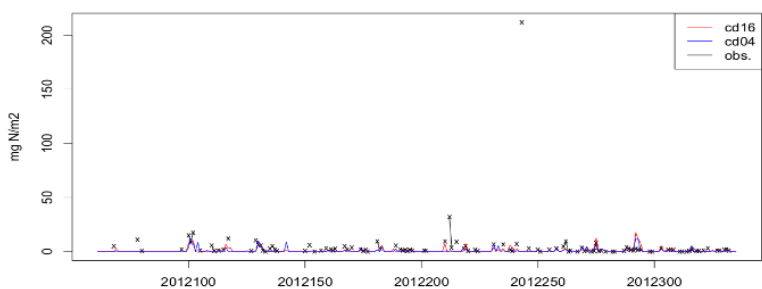

(d) Leba, PL0004R

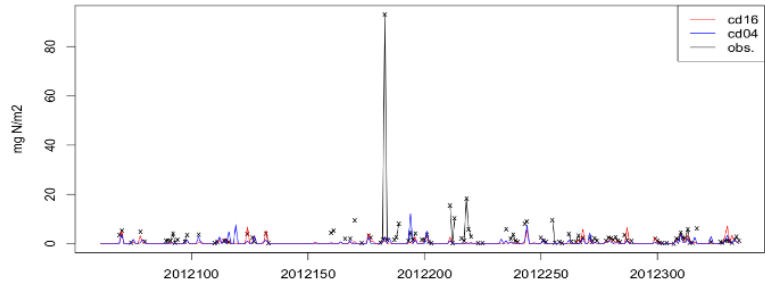

(f) Lahemaa, EE0009R

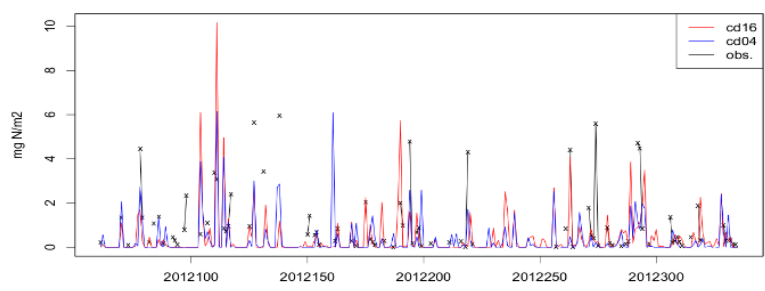

(h) Virolahti II, FI0017R

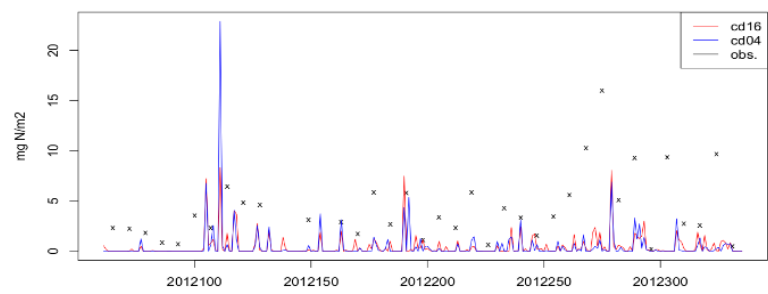

(j) Preila, LT0015R

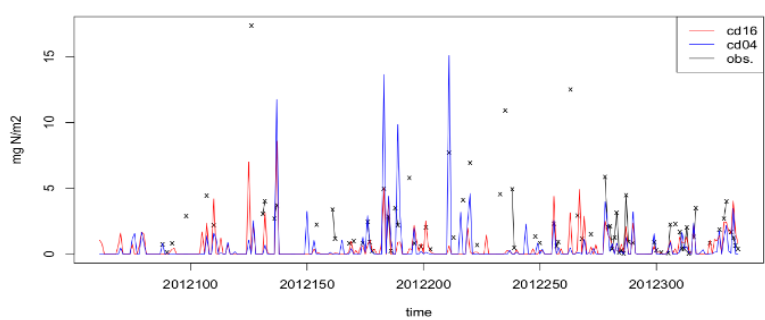

Figure S5. Comparison of modelled wet deposition of ammonium as daily sums $\left(\mathrm{mg}(\mathrm{N}) \mathrm{m}^{-2}\right.$ $\mathrm{d}^{-1}$ ) from the 16-km resolution grid (red) and 4km-resolution grid (blue) against observed daily sums of nitrate wet deposition (black crosses) at regional background stations around the Baltic Sea from the EMEP monitoring network: (a) Zingst, DE0009R, (b) Råö, SE0014R, (c) Diabla Gora, PL0005R, (d) Leba, PL0004R, (e) Ähtäri, FI0004R, (f) Lahemaa, EE0009R, (g) Hailuoto II, FI0053R, (h) Virolahti II, FI0017R, (i) Vilsandi, EE0011R, and (j) Preila, LT0015R. Stations correspond to those in Table 5. Comparison time period: 1 March to 30 November 2012. 
(a)

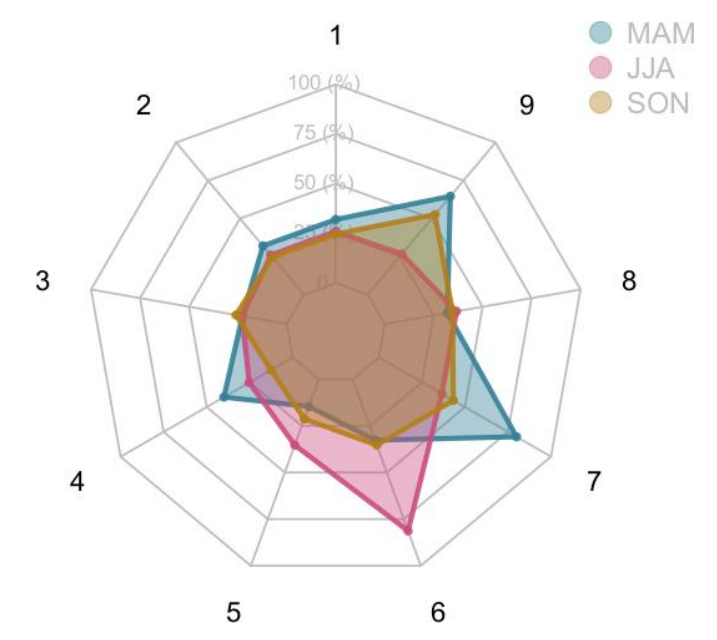

(b)

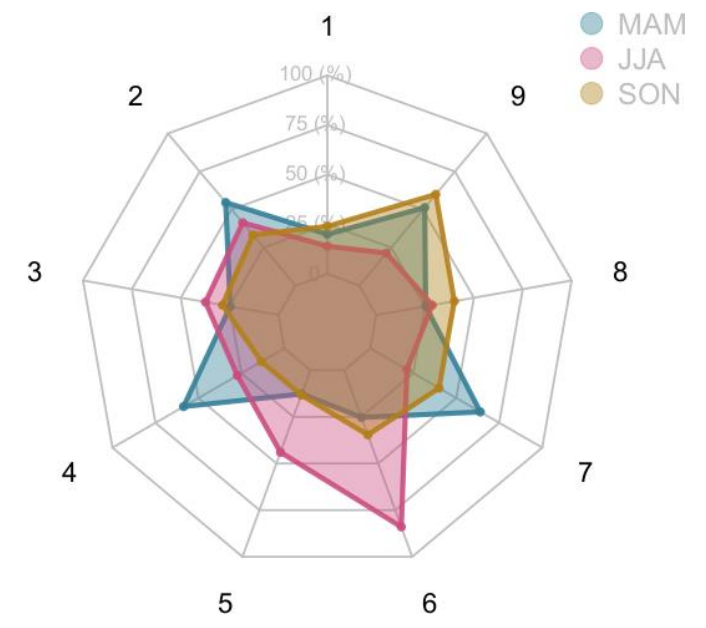

Figure S6. Spider charts of the ratio between modelled and observed seasonal averages: (a) wet deposition of nitrate and (b) wet deposition of ammonium, for spring (MAM), summer (JJA) and autumn (SON). Stations indicated by numbers 1: Zingst, DE0009R; 2: Lahemaa, EE0009R; 3: Ähtäri, FI0004R; 4: Virolahti II, FI0017R; 5: Hailuoto II, 6: Preila, LT0015R; 7: Leba, PL0004R; 8: Diabla Gora, PL0005R; 9: Råö, SE0014R. The maximum (100 \%) corresponds to a ratio of 1.5 . 
(a)

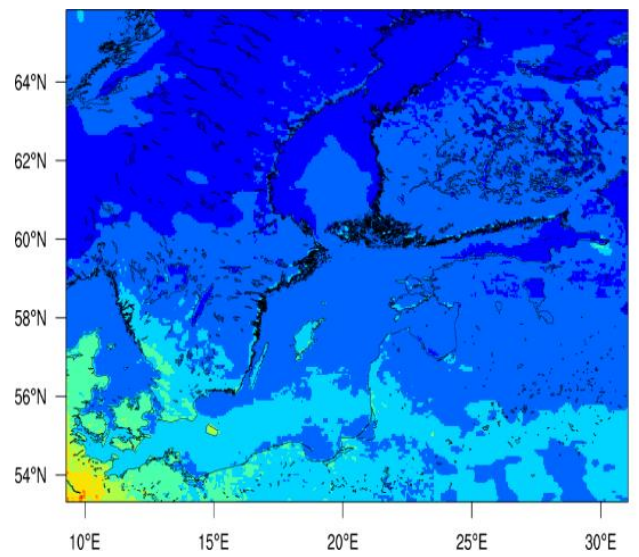

(c)

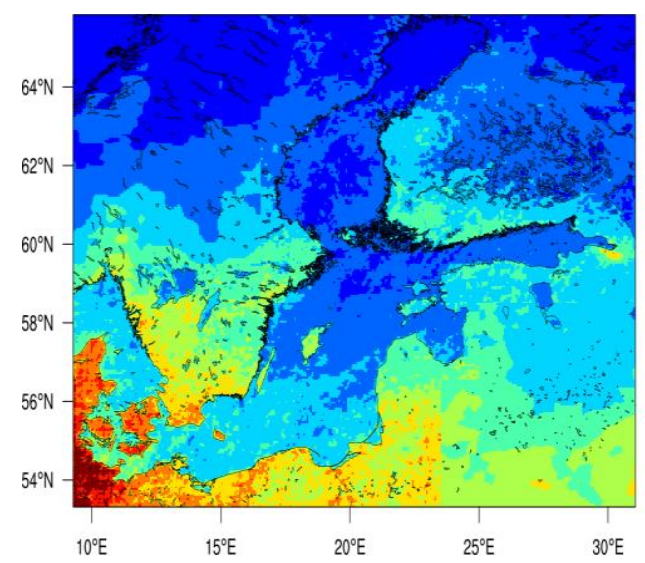

(b)

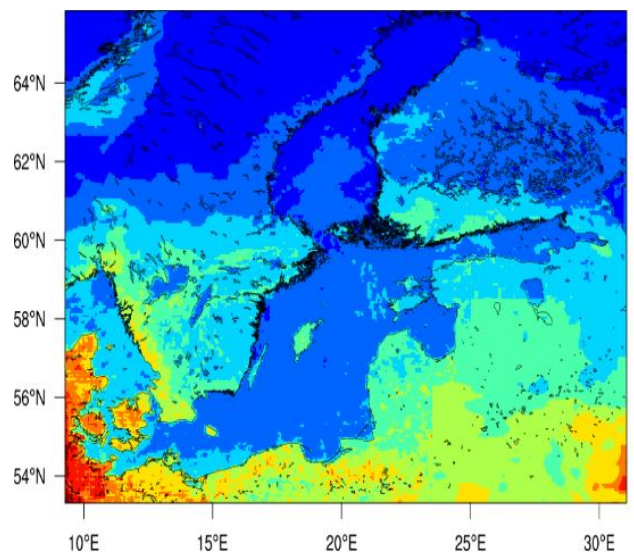

(d)

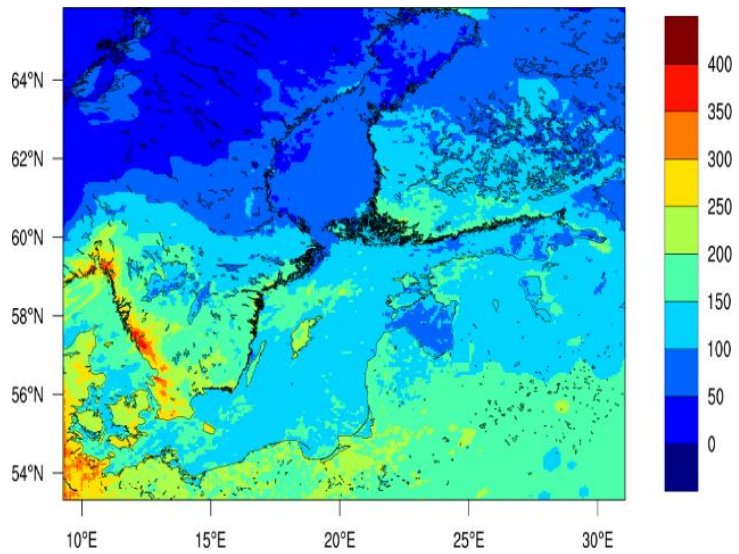

Figure S7. Present-day (2012) seasonal sums of total nitrogen deposition (in $\mathrm{mg}(\mathrm{N}) \mathrm{m}^{-2}$ ) from the CMAQ run with all emissions: (a) in winter (JFD), (b) in spring (MAM), (c) in summer (JJA), and (d) in autumn (SON). 
(a)

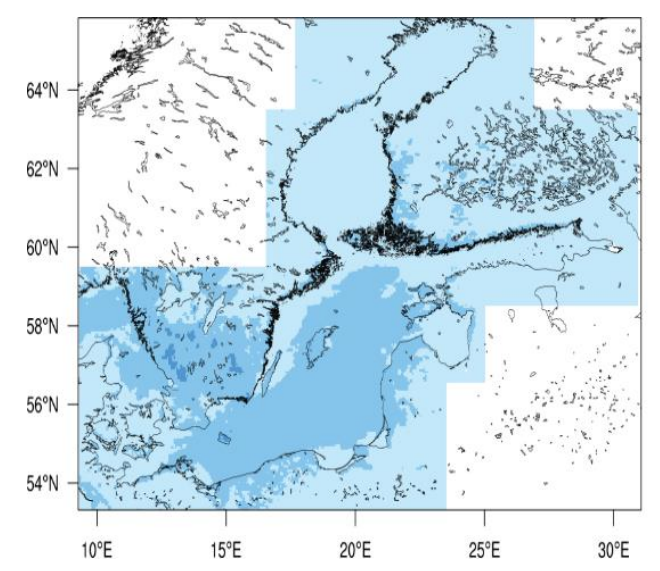

(c)

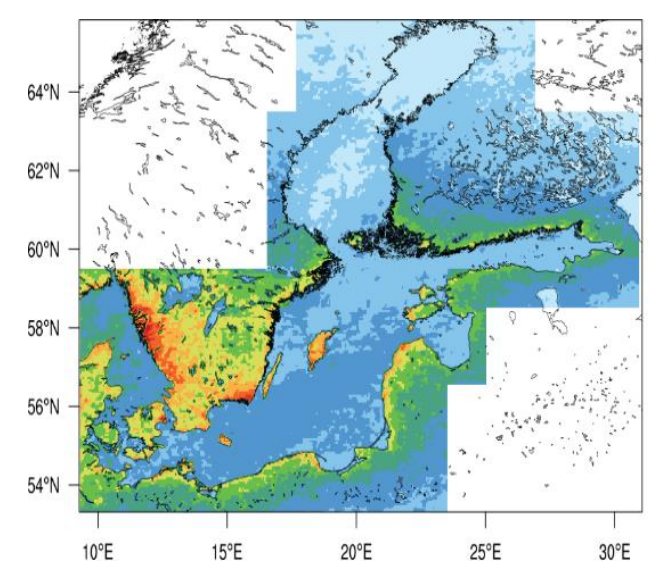

(b)

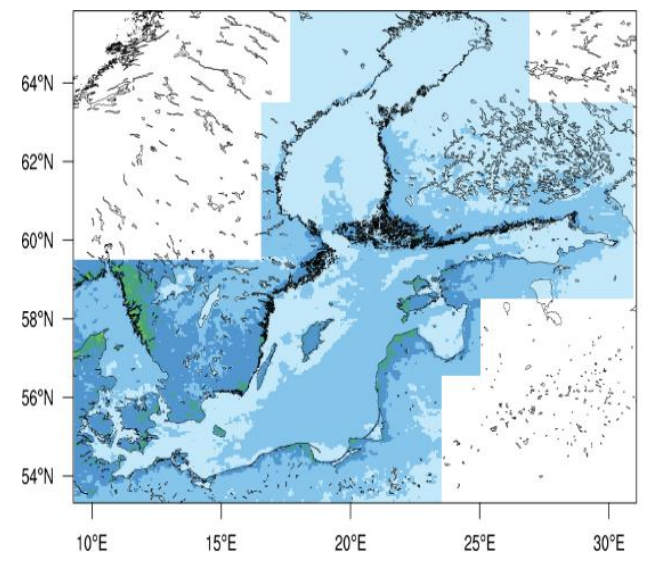

(d)

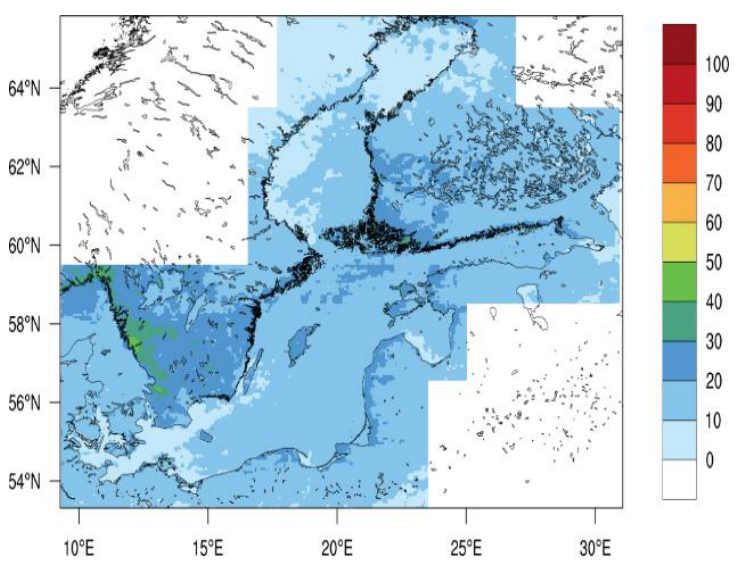

Figure S8. Present-day (2012) absolute ship contribution to the seasonal sums of nitrogen deposition (in $\mathrm{mg}(\mathrm{N}) \mathrm{m}^{-2}$ ): (a) in winter (JFD), (b) in spring (MAM), (c) in summer (JJA), and (d) in autumn (SON). Maps only show results for the high-resolution area. 
(a)

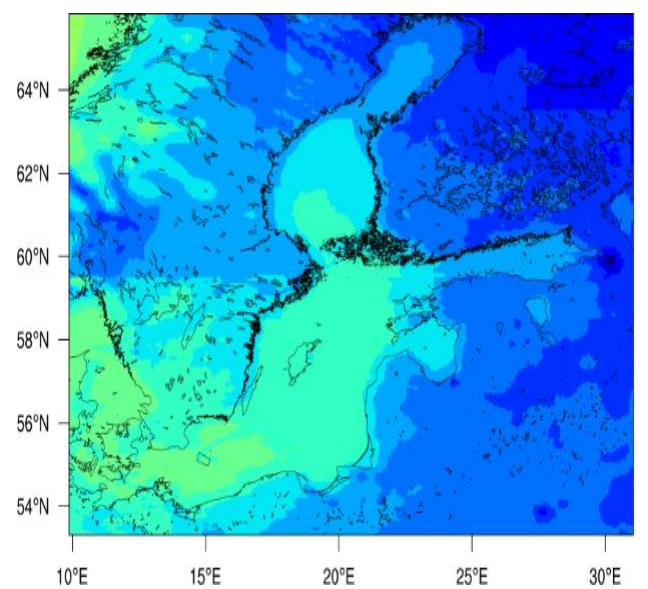

(c)

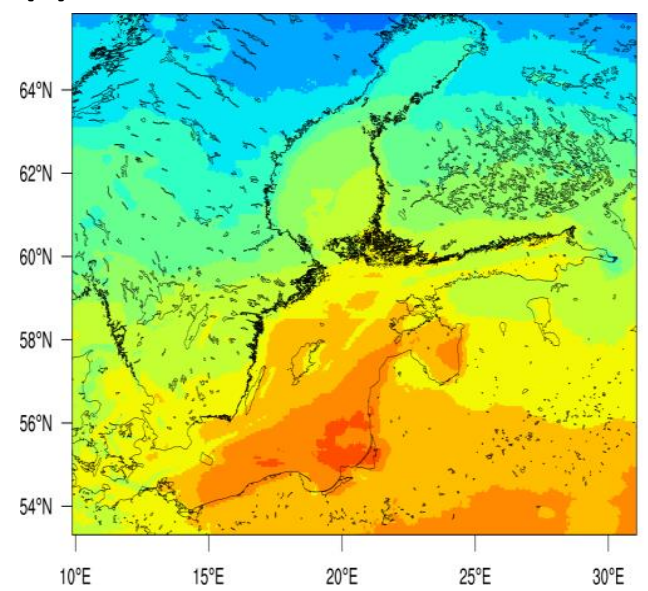

(b)

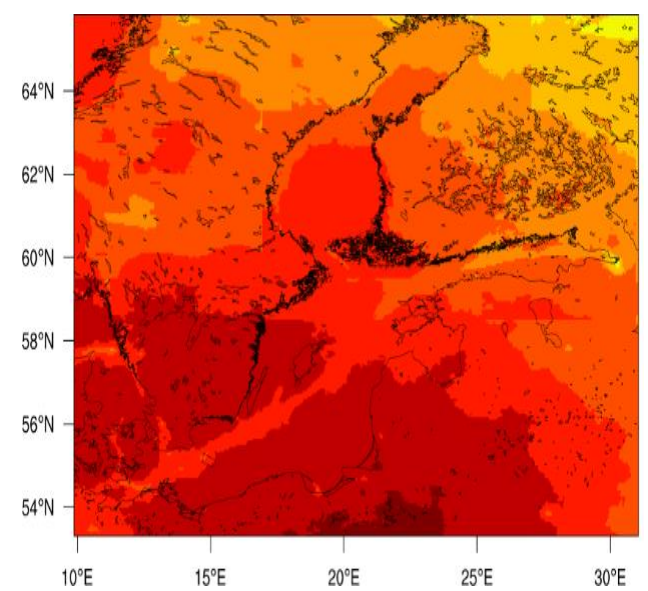

(d)

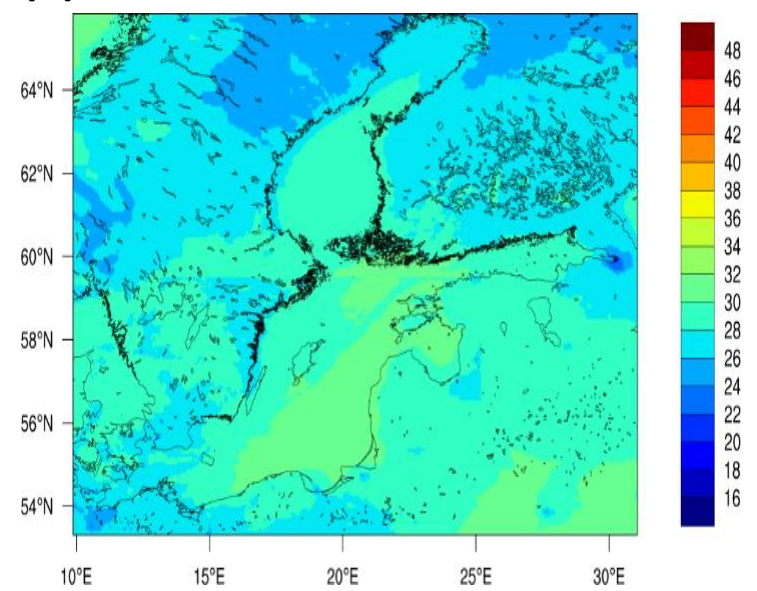

Figure S9. Present-day (2012) seasonal average of the daily maximum $\mathrm{O}_{3}$ concentration (in ppbv) in the Baltic Sea region from the CMAQ run with all emissions: (a) mean of winter months (JFD), (b) mean of spring months (MAM), (c) mean of summer months (JJA), and (d) mean of autumn months (SON). 
(a)

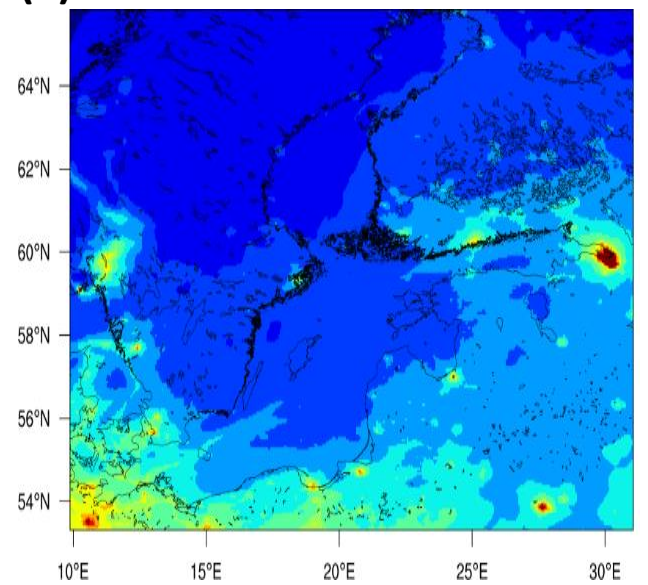

(c)

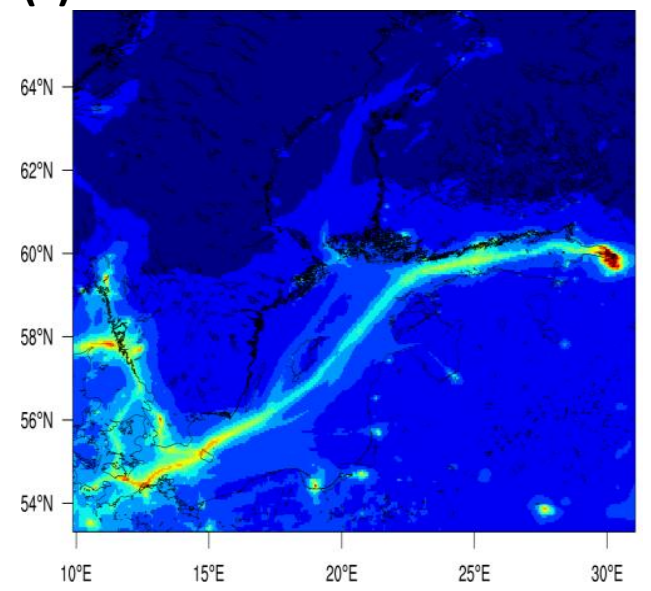

(b)

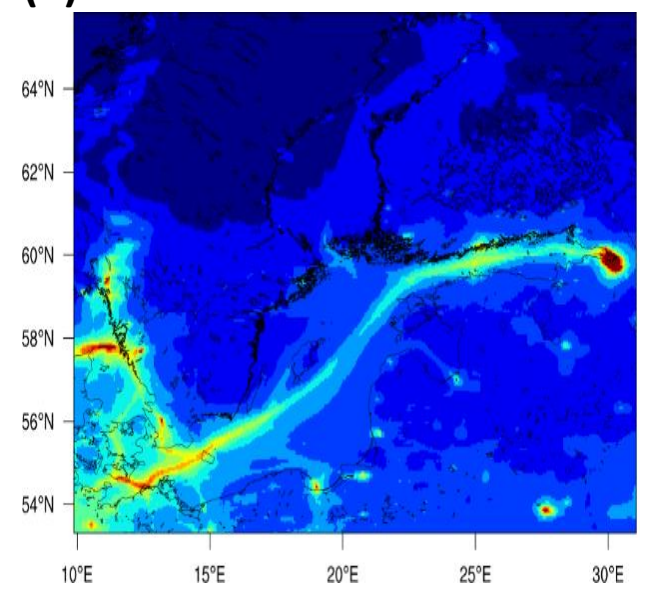

(d)

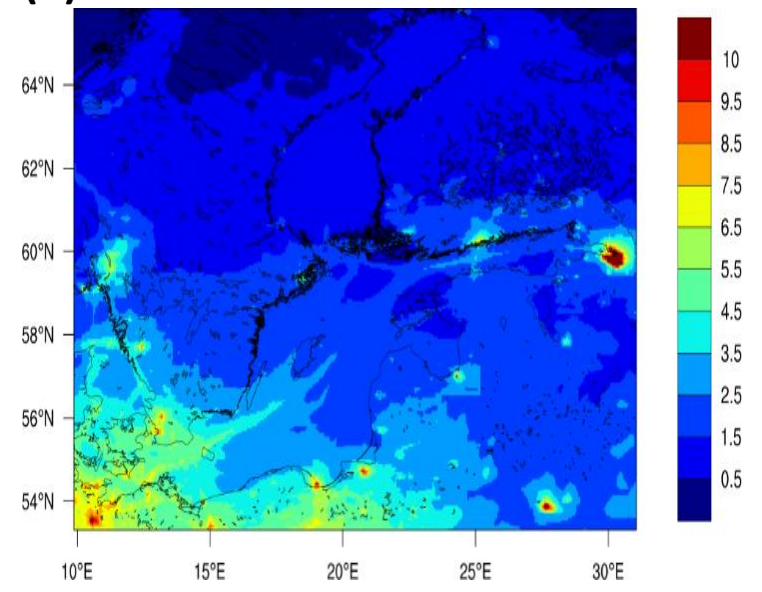

Figure S10. Present-day (2012) seasonal average concentration of $\mathrm{NO}_{2}$ (in ppbv) in the Baltic Sea region from the CMAQ run with all emissions: (a) mean of winter months (JFD), (b) mean of spring months (MAM), (c) mean of summer months (JJA), and (d) mean of autumn months $(\mathrm{SON})$. 
(a)

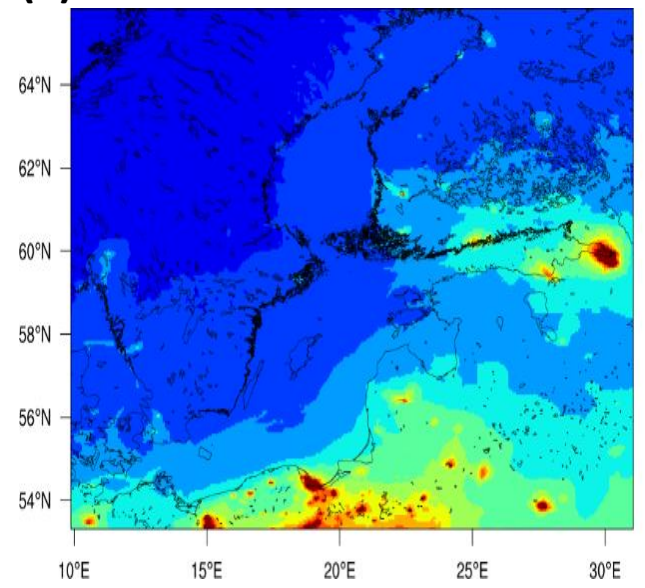

(c)

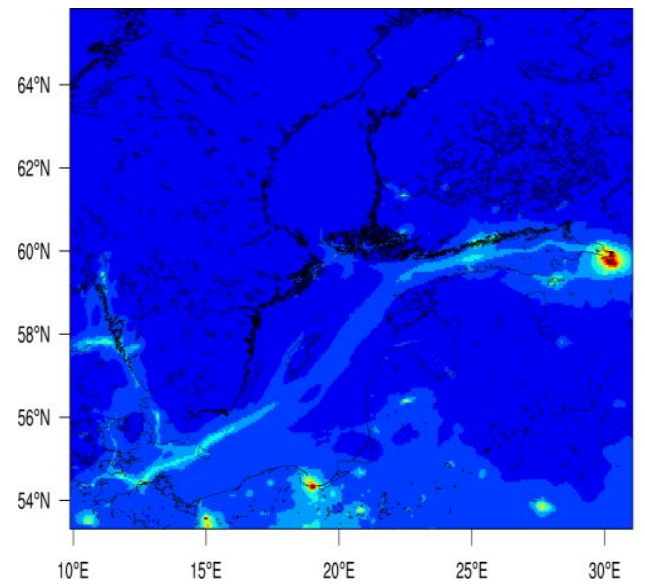

(b)

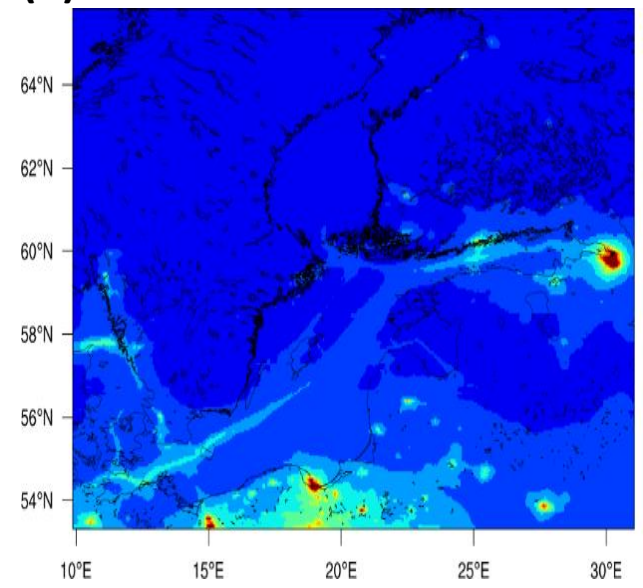

(d)

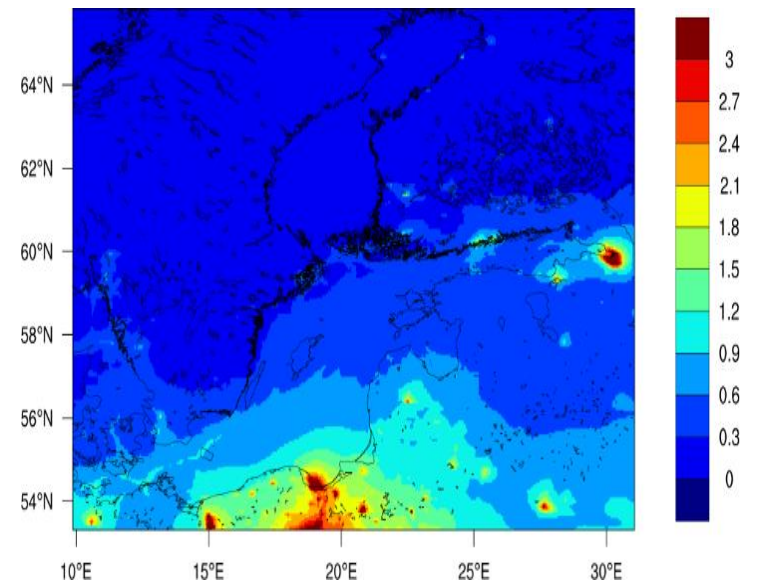

Figure S11. Present-day (2012) seasonal average concentration of $\mathrm{SO}_{2}$ (in ppbv) in the Baltic Sea region from the CMAQ run with all emissions: (a) mean of winter months (JFD), (b) mean of spring months (MAM), (c) mean of summer months (JJA), and (d) mean of autumn months (SON). 
(a)

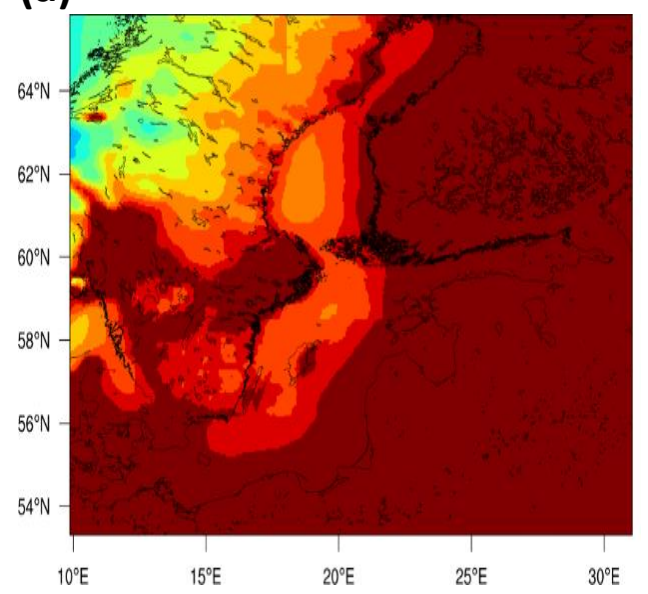

(c)

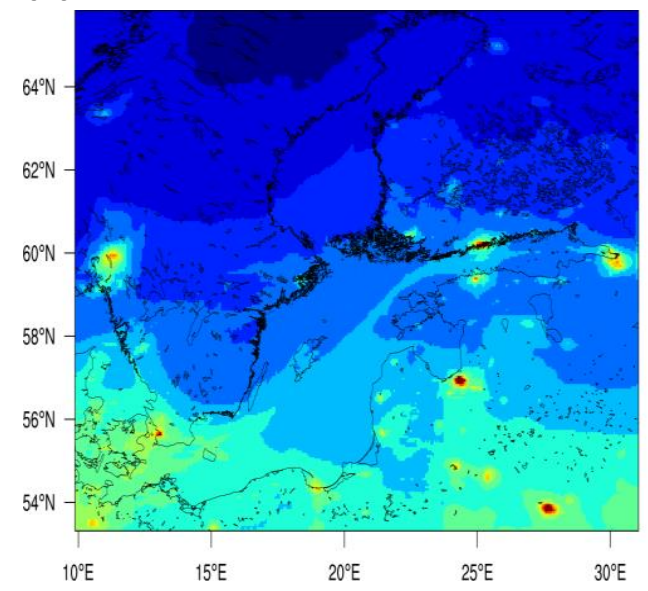

(b)

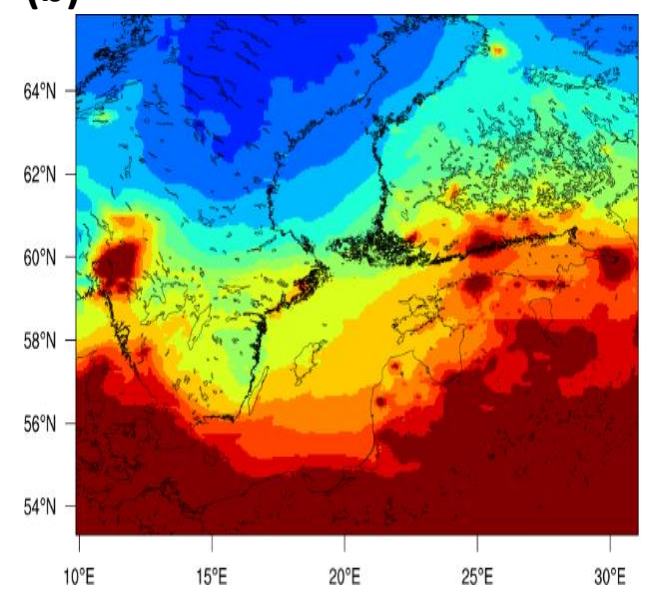

(d)

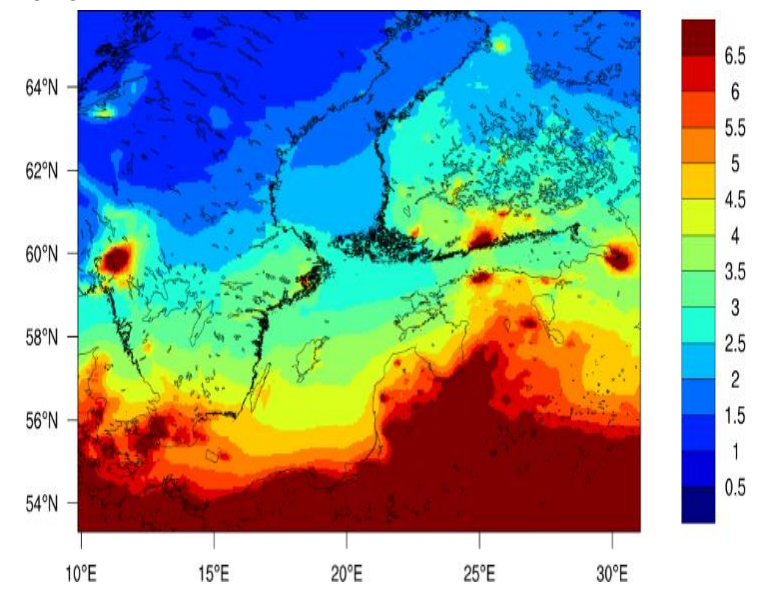

Figure S12. Present-day (2012) seasonal average concentration of PM2.5 (in $\mu \mathrm{g} \mathrm{m}^{-3}$ ) in the Baltic Sea region from the CMAQ run with all emissions: (a) mean of winter months (JFD), (b) mean of spring months (MAM), (c) mean of summer months (JJA), and (d) mean of autumn months (SON). 
(a)

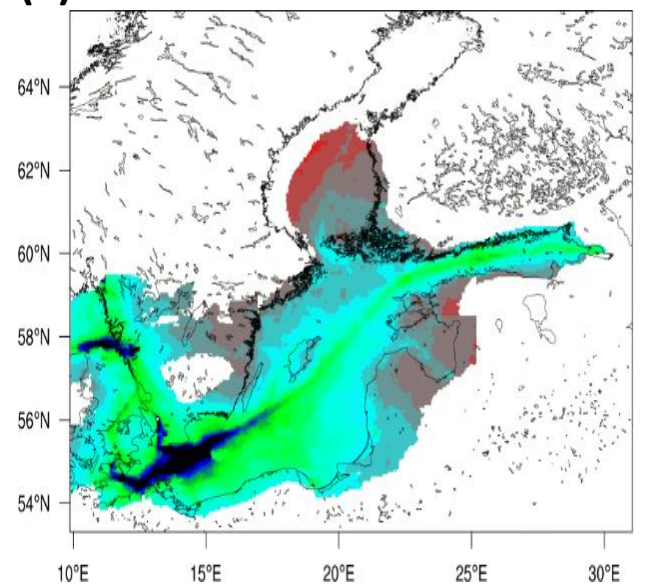

(c)

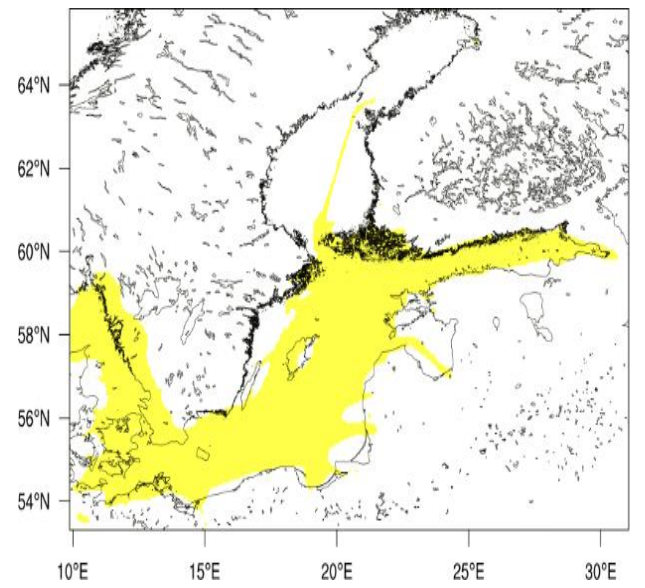

(b)
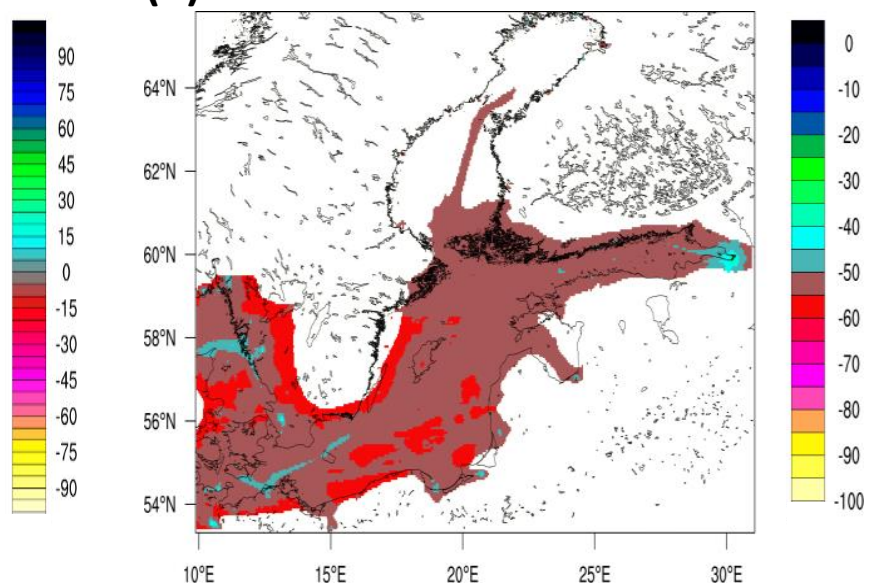

(d)
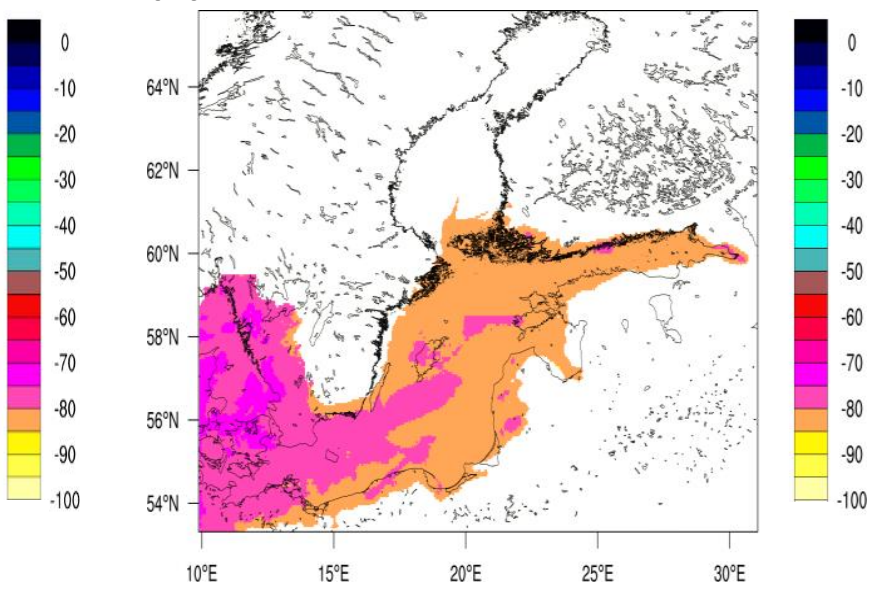

Figure S13. Future (2040) change of the ship-related contribution in summer (JJA) in percent compared to 2012, given as rel. difference between the ship contribution from the "NoNECA 2040" simulation and the ship contribution from the present-day simulation: (a) daily maximum $\mathrm{O}_{3}$, (b) $\mathrm{NO}_{2}$, (c) $\mathrm{SO}_{2}$, (d) $\mathrm{PM}_{2.5}$. Not coloured (empty) areas indicate grid cells with ship contribution in "BAU 2040" of less than $1.0 \mathrm{ppbv}, 0.1 \mathrm{ppbv}, 0.01 \mathrm{ppbv}, 0.005 \mu \mathrm{g} \mathrm{m}^{-3}$, for daily max. $\mathrm{O}_{3}, \mathrm{NO}_{2}, \mathrm{SO}_{2}, \mathrm{PM}_{2.5}$, respectively. Ship-related contribution only shown for the high-resolution area. Note the different scale for daily max. $\mathrm{O}_{3}$ (from $-100 \%$ to $100 \%$ ). 
(a)

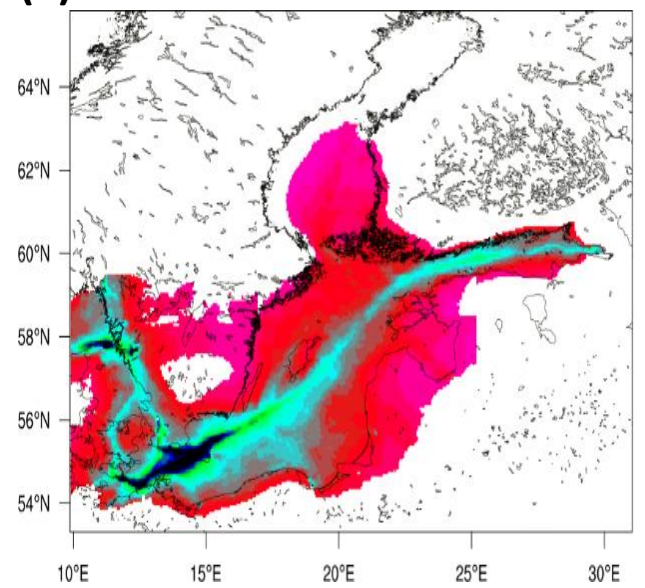

(c)

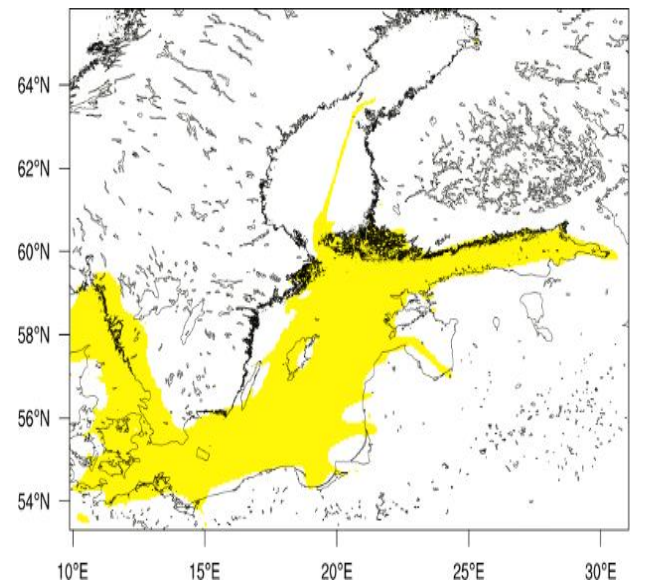

(b)
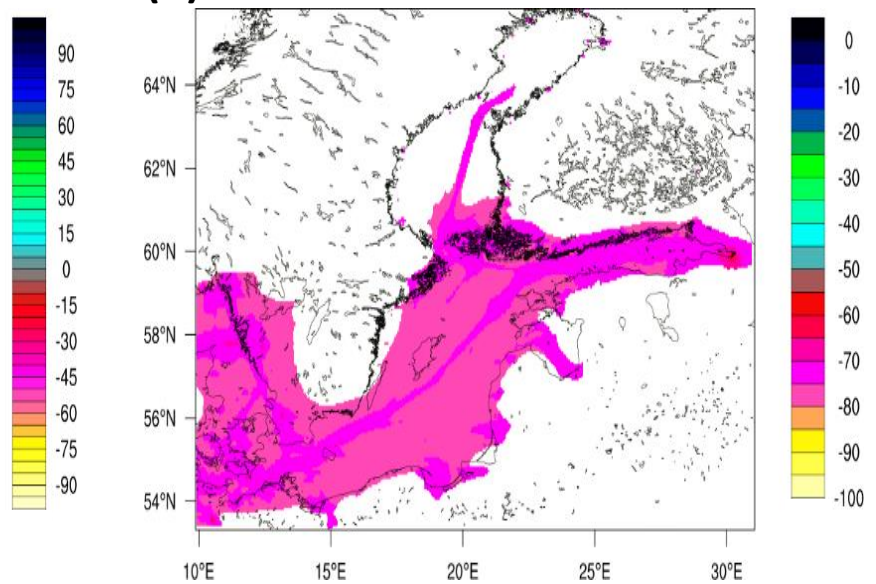

(d)
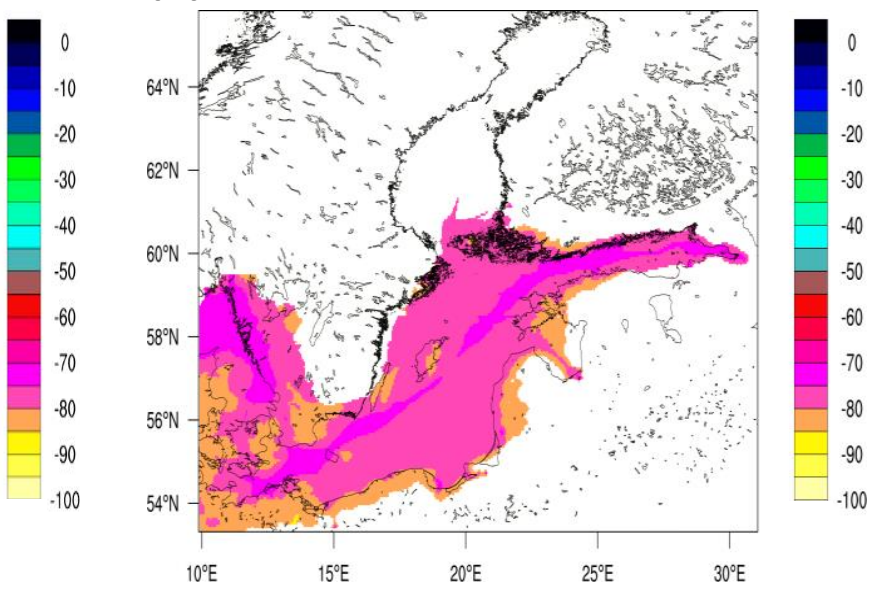

Figure S14. Future (2040) change of the ship-related contribution in summer (JJA) in percent compared to 2012, given as rel. difference between the ship contribution from the "EEDI 2040" simulation and the ship contribution from the present-day simulation: (a) daily maximum $\mathrm{O}_{3}$, (b) $\mathrm{NO}_{2}$, (c) $\mathrm{SO}_{2}$, (d) $\mathrm{PM}_{2.5}$. Not coloured (empty) areas indicate grid cells with ship contribution in "BAU 2040" of less than $1.0 \mathrm{ppbv}, 0.1 \mathrm{ppbv}, 0.01 \mathrm{ppbv}, 0.005 \mu \mathrm{g} \mathrm{m}^{-3}$, for daily max. $\mathrm{O}_{3}, \mathrm{NO}_{2}, \mathrm{SO}_{2}, \mathrm{PM}_{2.5}$, respectively. Ship-related contribution only shown for the high-resolution area. Note the different scale for daily max. $\mathrm{O}_{3}$ (from $-100 \%$ to $100 \%$ ). 
(a)

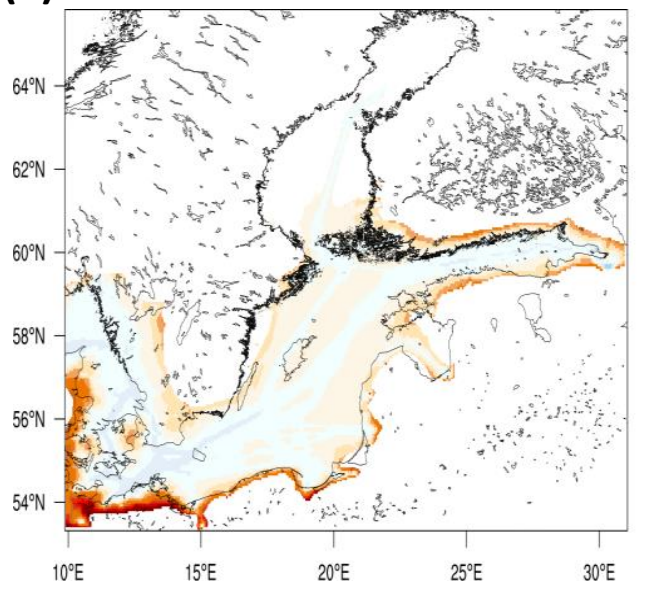

(b)

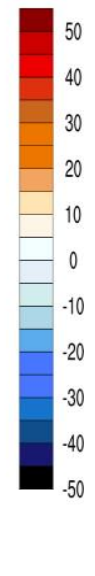

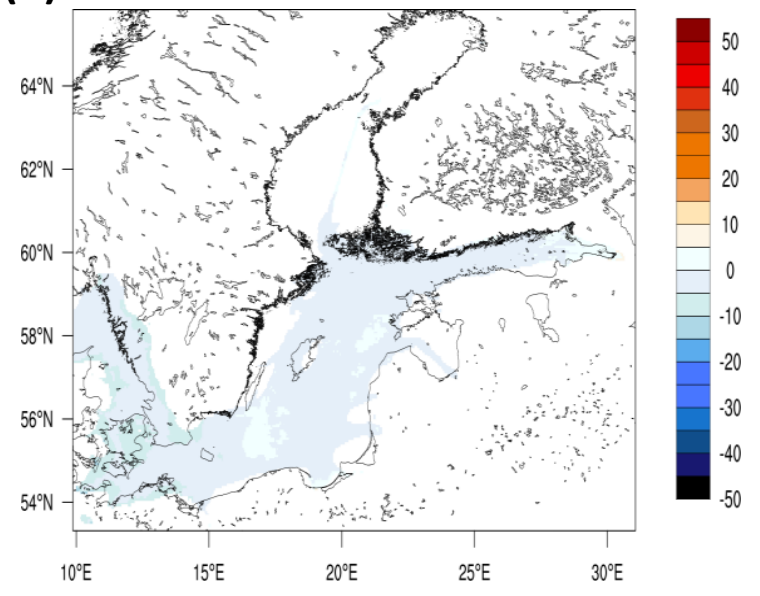

Figure S15. Effect of reduced land-based emissions (in percent) on the future ship contribution of (a) $\mathrm{NO}_{2}$ and (b) $\mathrm{SO}_{2}$ in summer (JJA) 2040 in the Baltic Sea region. Not coloured (white) areas indicate grid cells with ship contribution in the scenario "BAU 2040" of less than 0.1 ppbv for $\mathrm{NO}_{2}$ and less than $0.01 \mathrm{ppbv}$ for $\mathrm{SO}_{2}$. 


\section{References}

EMEP: Manual for sampling and chemical analysis. Kjeller, Norwegian Institute for Air Research (EMEP/CCC Report 1/95) (Last rev. February 2014). Available at: https://www.nilu.no/projects/ccc/manual/download/cccr1-95rev.pdf, (Last Accessed 07.10.2018), 2014.

Giordano, L., Brunner, D., Flemming, J., Hogrefe, C., Im, U., Bianconi, R., Badia, A., Balzarini, A., Baró, R., Chemel, C., Curci, G., Forkel, R., Jiménez-Guerrero, Hirtl, M., Hodzic, A., Honzak, L., Jorba, O., Knote, C., Kuenen, J. J. P., Makar, P. A., Manders-Groot, A., Neal, L., Pérez, J. L., Pirovano, G., Pouliot, G., San José, R., Savage, N., Schröder, W., Sokhi, R. S., Syrakov, D., Torian, A., Tuccella, P., Werhahn, J., Wolke, R., Yahya, K., Žabkar, R., Zhang, Y., and Galmarini, S., Assessment of the MACC reanalysis and its influence as chemical boundary conditions for regional air quality modeling in AQMEII-2, Atmos. Environ., 115, 371-388, doi:10.1016/j.atmosenv.2015.02.034, 2015.

Nespor, V. and Sevruk, B.: Estimation of wind-induced error of rainfall gauge measurements using a numerical simulation, J. Atmos. Oceanic Technol., 16, 450-464, 1998.

Tørseth, K., Aas, W., Breivik, K., Fjæraa, A. M., Hjellbrekke, A. G., Lund Myhre, C., Solberg, S., and Yttri, K. E.: Introduction to the European Monitoring and Evaluation Programme (EMEP) and observed atmospheric composition change during 1972-2009, Atmos. Chem. Phys., 12, 5447-5481, doi:10.5194/acp-12-5447-2012, 2012.

WMO: World Meteorological Organization, Climate Indices and Analysis for Sectoral Application, , paper presented by Leslie Malone at the Abdus Salam International Centre for Theoretical Physics (ICTP) Summer School on Climate Impacts Modeling for Developing Countries: Water, Agriculture and Health, 5-16 September 2011, Trieste, Italy, 2011. 